\title{
The Effect of a Hadley Circulation on the Propagation and Reflection of Planetary Waves in a Simple One-Layer Model
}

\author{
J. GAVIN ESLER \\ Program in Atmospheres, Oceans and Climate, Massachusetts Institute of Technology, Cambridge, Massachusetts \\ LoRENZO M. POLVANI \\ Department of Applied Physics and Applied Mathematics, Columbia University, New York, New York \\ R. Alan Plumb \\ Program in Atmospheres, Oceans and Climate, Massachusetts Institute of Technology, Cambridge, Massachusetts
}

(Manuscript received 14 December 1998, in final form 30 June 1999)

\section{ABSTRACT}

\begin{abstract}
The effect of a simple representation of the Hadley circulation on the propagation and nonlinear reflection of planetary-scale Rossby waves in the winter hemisphere is investigated numerically in a single-layer shallowwater model.

In the first instance, waves are forced by a zonal wavenumber three topography centered in the extratropics. In the linear limit the location of the low-latitude critical line at which the waves are absorbed is displaced poleward by the Hadley circulation. At finite forcing amplitude the critical layer regions where the waves break are found to be displaced poleward by a similar distance. The Hadley circulation is also found to inhibit the onset of nonlinear reflection by increasing the dissipation of wave activity in the critical layer.

Second, for waves generated by an isolated mountain, the presence of the Hadley circulation further inhibits nonlinear reflection by generating a strong westerly flux of wave activity within the critical layer. This westerly flux is shown to be largely advective and is explained by the poleward displacement of the critical line into the region of westerly flow. A simple expression is derived for the minimum zonal wind strength allowing propagation in the case of a quasigeostrophic $\beta$-plane flow when the mean meridional wind $\bar{v}>0$.
\end{abstract}

\section{Introduction}

A great deal of effort has been expended in theoretical studies aimed at explaining the observed stationary wave response of the atmosphere to orographic forcing, and observed teleconnection patterns; see, for example, the review by Frederiksen and Webster (1988). One of the most fundamental approaches is to linearize the equations of motion about an axisymmetric flow and investigate the response to an isolated forcing (Hoskins et al. 1977; Grose and Hoskins 1979; Hoskins and Karoly 1981; Webster 1981). These studies, and others, showed that planetary-scale Rossby waves tend to propagate approximately along the arcs of great circles. However, linear theory does not give insight into the behavior of finite-amplitude wave trains when they

Corresponding author address: Dr. J. G. Esler, Centre for Atmospheric Science, Department of Applied Mathematics and Theoretical Physics, University of Cambridge, Silver Street, Cambridge CB3 9EW, United Kingdom.

E-mail: jge1000@amtp.cam.ac.uk reach a critical line, where the flow speed equals the phase speed of the waves (e.g., Killworth and McIntyre 1985).

Recently, Waugh et al. (1994, hereafter WPP) and Brunet and Haynes (1996, hereafter BH), have used a single-layer model to investigate the nonlinear behavior of finite-amplitude stationary waves as they approach the zero wind line in low latitudes. Both studies showed a strong tendency for the wave train to be reflected back into midlatitudes from the longitude where the waves begin to break. Similar results have been also been obtained in a realistic three-dimensional flow by Magnusdottir and Haynes (1999). It is certainly an open question as to whether such reflection can be observed in the atmosphere, but typical pseudomomentum flux analyses of stationary wave patterns (e.g., Karoly et al. 1989) seem to indicate that waves often propagate from the extratropics and are absorbed at low latitudes, at least in the case when the flow there is easterly. Both WPP and BH emphasize the fact that their respective models did not contain a representation of the Hadley circulation, which might change the behavior of the low- 
latitude critical layer from reflecting to absorbing. In this paper we aim to address this issue, by comparing the behavior of waves excited in simple single-layer flows that are closely comparable except for the presence or absence of a representation of the Hadley circulation. Partly because of the limitations of the model chosen, we concentrate on the winter hemisphere case.

There have been several studies of the effect of the Hadley circulation on linear wave propagation characteristics. Schneider and Watterson (1984) showed that southward linear propagation across an easterly layer is possible in a shallow-water model provided the mean meridional flow across the layer is also to the south. They also developed an analysis of the quasigeotrophic $\beta$-plane case that was further extended by Farrell and Watterson (1985). They showed that when the mean meridional flow was to the north, the critical line "barrier" to propagation was shifted to the north (see also the discussion in section 4 a below). Finally, a threedimensional primitive-equation model was studied by Watterson and Schneider (1987), who showed that a Hadley circulation could allow cross-hemispheric propagation from the summer hemisphere in a more realistic flow.

Held and Phillips (1990, hereafter HP) are the only authors who, to our knowledge, have investigated the nonlinear interaction of the Hadley circulation and Rossby wave breaking. They used a hybrid model in which a zonally periodic, barotropic Rossby wave encounters the mean meridional flow generated in a zonally averaged, forced shallow-water system with a layer depth of $1 \mathrm{~km}$. This value is chosen as an appropriate equivalent depth for the upper-tropospheric branch of the Hadley circulation, when the effects of moisture are taken into account. Although they were motivated primarily by the need to understand the zonal mean angular momentum budget, their results seem to indicate that significant long-term, low-latitude absorption of finiteamplitude waves can be maintained, in contrast to the situation when the low-latitude critical layer is entirely inviscid.

Based on these earlier results, two key questions to be addressed in this study are the following.

1) What effect, if any, does the shift in the position of the "barrier" due to the Hadley circulation have on the propagation, breaking, and nonlinear reflection of finite-amplitude waves?

2) To what extent does the Hadley circulation inhibit the nonlinear reflection observed in "no-Hadley circulation" experiments and allow prolonged absorption of incoming wave activity?

In section 2 we describe the single-layer shallowmodel used for our experiments. The model can be thought to represent an isentropic layer spanning the upper troposphere-and some advantages and limitations of this view are described in HP. Following BH, in order to use a single consistent model for our ex- periments, we choose a compromise layer depth $(5 \mathrm{~km})$ between that thought appropriate for extratropical barotropic Rossby waves $(\approx 7-10 \mathrm{~km})$ and that suggested by HP for the Hadley circulation $(1 \mathrm{~km})$. Although this choice has its disadvantages, discussed below, we believe that the advantages conferred by the simplicity of our model, together with the fact that direct dissipation of the eddies by this forcing is shown not to be the most important component in the wave activity budgets, justify such an approach. In section $3 \mathrm{a}$ we describe experiments in which the waves are forced by a zonally periodic wave-3 topography (as in HP), while in section $3 \mathrm{~b}$ we use an isolated mountain to generate solitary wave trains (as in WPP and BH). In the discussion of section $4 \mathrm{a}$ we revisit the linear theory of wave propagation in the presence of a mean meridional current by reviewing the quasigeostrophic $\beta$-channel analysis of Schneider and Watterson (1984). Wave activity budgets are then calculated for both the zonally symmetric wave-3 experiments and the isolated mountain experiments in section $4 \mathrm{~b}$. In section 5 the effect of the presence of the Hadley circulation on the time mean stationary wave structure is investigated, along with its effect on the Simmons-Wallace-Branstator instabilities reported for this type of model by Polvani et al. (1999). The summary and conclusions are presented in section 6 .

\section{The model and experiments}

The physical model used in this paper is an incompressible one-layer fluid of mean depth $H=5 \mathrm{~km}$, obeying the nondimensional forced shallow-water equations:

$$
\begin{array}{r}
D_{t} \mathbf{v}+\mathbf{k} \times \mathbf{v}+(\mathrm{Fr})^{-1} \nabla\left(\sigma+h_{T}\right)=\mathbf{G} \\
D_{t} \sigma+\sigma \boldsymbol{\nabla} \cdot \mathbf{v}=Q,
\end{array}
$$

where $\sigma$ is the thickness of the fluid layer, $h_{T}$ is the bottom topography, $\mathbf{v}=(u, v)$ is the two-dimensional velocity vector, and $D_{t} \equiv \partial_{t}+\mathbf{v} \cdot \boldsymbol{\nabla}$ is the material derivative. Here, $\mathrm{Fr}=\Omega^{2} a^{2} / g H=4.416$ is the Froude number in terms of the rotation rate $\Omega$, radius $a$, gravity $g$, and the layer depth $H$. The vector $\mathbf{G}=(F, G)$ and $Q$ represent a momentum forcing and a mass source or heating term, respectively. These equations are numerically integrated using a standard pseudospectral method (Hack and Jacob 1992). The computations reported below are performed using a T85 truncation (corresponding to a Gaussian grid with approximately $1.4^{\circ}$ grid spacing), and a standard $\nabla^{6}$ hyperdissipation on the left side of (1) acts on both the thickness and velocity fields. The hyperdiffusivity $\nu$ is adjusted to the lowest possible value consistent with numerical stability in each experiment. The value used, $4 \times 10^{-12} a^{6} \Omega$, corresponds approximately to a 2.5 -h $e$-folding scale for the smallest resolved scale $n=85$. 


\section{a. Experiments with a Hadley circulation}

For these experiments we generate a shallow-water Hadley circulation by choosing

$$
\begin{aligned}
Q & =-\kappa_{T}\left(\bar{\sigma}-\sigma_{\mathrm{eq}}\right) \\
\mathbf{G} & =-\left[\kappa_{M}+\kappa_{T} \frac{\left(\sigma_{\mathrm{eq}}-\bar{\sigma}\right)}{\bar{\sigma}} \mathcal{H}\left(\sigma_{\mathrm{eq}}-\bar{\sigma}\right)\right] \overline{\mathbf{v}},
\end{aligned}
$$

where $\boldsymbol{\kappa}_{T}^{-1}$ and $\boldsymbol{\kappa}_{M}^{-1}$ are thermal and momentum relaxation timescales, respectively; and $\sigma_{\text {eq }}$ is an equilibrium layer thickness, chosen in order to generate a reasonable northern winter Hadley circulation. The Heaviside function $\mathcal{H}$ is defined so that $\mathcal{H}(x)=1$ when $x>0$ and is zero otherwise, and overbars denote zonal mean quantities. In (2) zonal means are taken to prevent the forcing terms from dissipating the eddy fields directly during the experiments, except where they project unto the zonal mean. (Eddy quantities are defined in this paper as deviations from a constant basic state, rather than the zonal mean.) This form of damping is chosen in order that there is as little difference as possible between the two sets of experiments in the dissipation of the eddies, so that any difference between pairs of experiments are as far as possible due only to the presence or absence of the Hadley circulation. ${ }^{1}$ The second term in the expression for the momentum forcing represents the drag due to the fluid mass source, which is assumed to be adding fluid with zero momentum from a notional boundary layer beneath the fluid layer that we are considering (cf. Fig. 1 in HP).

If the equations (1) are integrated for 10-20 relaxation timescales $\approx \max \left\{\kappa_{T}^{-1}, \kappa_{M}^{-1}\right\}$ from a balanced, zonally symmetric initial state, a steady, zonally symmetric Hadley circulation state results. The nature of this basic state depends on the parameters $\kappa_{T}$ and $\kappa_{M}$, as well as the equilibrium height field $\sigma_{\text {eq }}$. Although in our singlelayer system the mean depth $(5 \mathrm{~km})$ is not close to the equivalent depth for the Hadley circulation in the tropical upper troposphere (HP suggest around $1 \mathrm{~km}$ ), we find that a Hadley cell of suitable strength compared with observations (see, e.g., Lindzen and Hou 1988), together with reasonable zonal mean winds, is generated by choosing $\kappa_{T}^{-1}=5$ days and $\kappa_{M}^{-1}=10$ days.

These values of the damping coefficients generate a circulation that is far from nonlinear, a nonlinear circulation in this sense being one associated with a region in the Tropics of constant angular momentum, and therefore zero potential vorticity. Although the zonal mean angular momentum field is not close to being constant in the tropical atmosphere, Held and Hou (1980) have shown that a nonlinear circulation is generated in an

\footnotetext{
${ }^{1}$ However, Magnusdottir and Haynes (1999) have recently shown that nonlinear reflection is not inhibited when thermal damping with a timescale as low as 5 days is acting on the waves, in the context of more realistic three-dimensional flows.
}

inviscid, axisymmetric atmosphere in the absence of any wave forcing due to either transients or stationary waves. One way to justify the high value of momentum drag in our model, and thus the dissipative nature of our Hadley circulation, is to consider the momentum drag to be a crude parameterization of the effects of transients propagating from the lower troposphere, rather than, for example, surface friction. (By experimenting with shallower layer depths, one might generate a more nonlinear circulation, but in our model this would be at the expense of having less realistic Rossby wave propagation from the extratropics.)

The wave activity budget of section $4 \mathrm{~b}$ below shows that direct dissipation of the zonal mean part of the eddies by the damping terms does not prove to be an important difference between experiments in which the Hadley circulation is present and absent. This means that from the point of view of wave propagation, one need not be too concerned as to whether the damping values can be justified for the upper troposphere.

The Hadley circulation basic state, denoted by $u_{0}^{H}$, $\boldsymbol{v}_{0}^{H}$, and $\sigma_{0}^{H}$, that results from the above process is then used as an initial state for the experiments to be described in section 3. In Fig. 1 the basic-state quantities are all plotted, along with the basic-state potential vorticity (PV hereafter) $P_{0}^{H}=\zeta_{a 0}^{H} / \sigma_{0}^{H}$, where $\zeta_{a 0}^{H}=2 \sin \varphi$ - $(\cos \varphi)^{-1}\left(u_{0}^{H} \cos \varphi\right)_{\varphi}$ is the basic-state absolute vorticity.

\section{b. Experiments without a Hadley circulation}

For these experiments we reproduce as closely as possible the initial conditions of the Hadley circulation experiments above, so that a close comparison can be made between the cases with Hadley circulation present and absent. We therefore take the basic-state zonal wind for these experiments to be $u_{0}^{N}=u_{0}^{H}$ defined above, and suppress the Hadley circulation by setting $\boldsymbol{v}_{0}^{N}=0$. If we consider the nonlinear balance equation given by

$$
(\mathrm{Fr})^{-1} \sigma_{0 \varphi}=-v_{0} v_{0 \varphi}-u_{0}\left(2 \sin \varphi+u_{0} \tan \varphi\right)+G_{0},
$$

the height field $\sigma_{0}^{N}$ can then be obtained by integration in latitude with $v_{0}=G_{0}=0$. This balance equation is also satisfied in the steady-state Hadley circulation case above, and as $|\bar{v}| \ll|\bar{u}|$, the initial thickness $\sigma_{0}^{N}$ for these experiments is almost identical to $\sigma_{0}^{H}$, as seen in Fig. 1b. As one would expect the PV gradient to be important in determining the refractive properties of the flow, we also show the PV profiles for the two experiments in Fig. 1d, along with a curve showing 100 times the difference between them, emphasizing the point that they are almost identical.

The forcing given by (2) for the Hadley circulation experiment is replaced by

$$
Q=0, \quad \mathbf{G}=-\kappa_{M}\left(\overline{\mathbf{v}}-\mathbf{v}_{0}^{N}\right),
$$

where $\mathbf{v}_{0}^{N}=\left(u_{0}^{N}, v_{0}^{N}\right)$, for the duration of each of the 

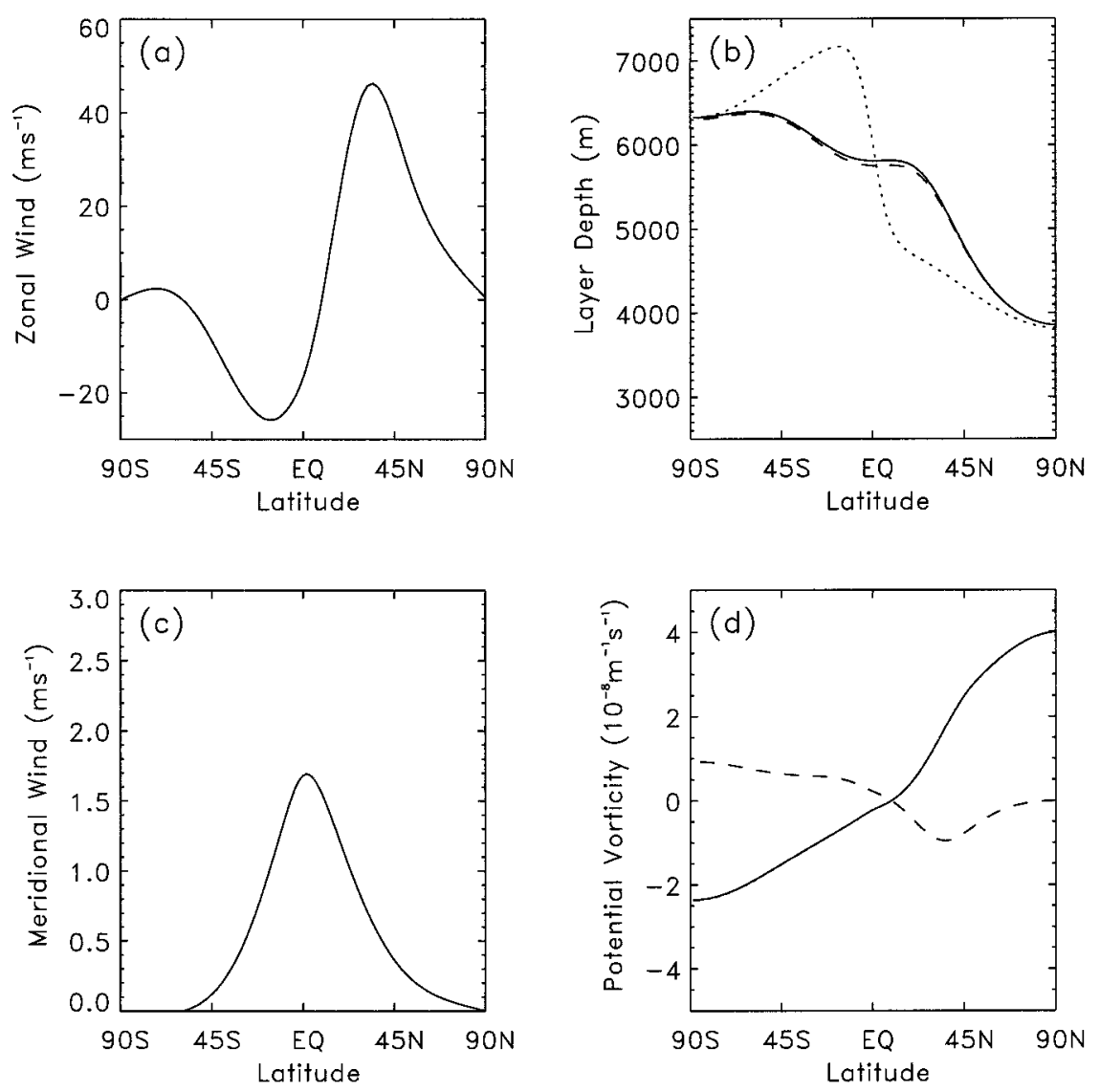

FIG. 1. The shallow-water basic-state circulations used in the experiments. (a) Zonal mean zonal wind $u_{0}^{H}\left(=u_{0}^{N}\right)$. (b) Zonal mean thickness $\sigma_{0}^{H}$ (solid curve), $\sigma_{0}^{N}$ (dashed curve), and radiative equilibrium height $\sigma^{\text {eq }}$ (dotted curve). (c) Meridional wind $v_{0}^{H}$. (d) Potential vorticity $P_{0}^{H}$ (solid curve) and $\left(P_{0}^{H}-P_{0}^{N}\right) \times 100$ (dashed curve).

experiments. The friction is retained so that the same friction acts directly on the zonal mean part of the eddies in the no Hadley circulation experiment as in the Hadley circulation experiment, despite there being no friction acting on the basic state.

\section{c. Topographic forcing}

Each experiment is integrated for 40 days, with initial basic-state conditions (hereafter $\mathbf{v}_{0}, \sigma_{0}$ ) taken to be $\mathbf{v}_{0}^{H}, \sigma_{0}^{H}$ when the Hadley cell is present and $\mathbf{v}_{0}^{N}, \sigma_{0}^{N}$ when it is absent. The equations are forced by (2) when the Hadley circulation is present and by (4) if it is not. In all the experiments the waves are generated by topography centered in the extratropics - see (1) - that is given the form $h_{T}=\epsilon F_{T}(\lambda, \varphi)\left(1-e^{-t / \tau}\right)$. Here, $\tau=5$ days is the timescale for the growth of the topography at the beginning of the experiment. The ratio of the height of the topography to the layer depth $H$ is given by $\epsilon$, which is used as a nondimensional measure of the wave-forcing amplitude in all that follows. In the case of the wave- 3 experiments described in section $3 \mathrm{a}$ (cf. HP),

$$
F_{T}(\lambda, \varphi)=\sin 3 \lambda e^{-\left[\left(\varphi-\varphi_{0}\right) / \varphi_{F}\right]^{2}},
$$

where the central latitude $\varphi_{0}=45^{\circ} \mathrm{N}$, and the decay scale of the topography is taken to be $\varphi_{F}=10^{\circ}$. In the case of the Gaussian mountain experiments described in section $3 \mathrm{~b}$ (cf. WPP), $F_{T}=F_{T}^{M}-\overline{F_{T}^{M}}$, where

$$
F_{T}^{M}(\lambda, \varphi)=e^{-\left(\vartheta / \vartheta_{F}\right)^{2}}
$$

where $\vartheta$ is the angle between the point $(\lambda, \varphi)$ and the top of the mountain. The mountain is centered at $45^{\circ} \mathrm{N}$, $90^{\circ} \mathrm{W}$, and the decay scale is again taken to be $\vartheta_{F}=$ $10^{\circ}$. The contribution to the zonal mean height is subtracted out at each latitude to avoid topographic forcing of the nonlinear balance equation (3). Note that this does not affect the amplitude of the Rossby waves generated in the linear limit.

\section{d. Wave activity diagnostics}

The wave activity relation used here to diagnose the experiments is essentially the shallow-water version of the generalized forced-dissipative relation of Haynes (1988), which was derived for the three-dimensional 
primitive equations. This version of the relation was presented in $\mathrm{BH}$, but without most of the source-sink terms given here, and the reader is referred to $\mathrm{BH}$ for a discussion of its general properties. The relation, which is exact for finite-amplitude waves, has the form

$$
\frac{\partial A}{\partial t}+\nabla \cdot \mathbf{F}=\mathcal{D}
$$

If the flow quantities, including the potential vorticity $P$, are divided into basic-state (e.g., $u_{0}, v_{0}, \sigma_{0}, P_{0}, \ldots$ ) and disturbance components $\left(u_{e}, v_{e}, \sigma_{e}, P_{e}, \ldots\right)$, then the (angular pseudomomentum) density $A$ is given by ${ }^{2}$

$$
A=\sigma X-\sigma_{e} u_{e} \cos \varphi
$$

where the integral

$$
X\left(P_{0}, P_{e}\right)=\int_{0}^{P_{e}}\left(P_{e}-\tilde{P}\right) \frac{d}{d \tilde{P}} m_{0}\left(P_{0}+\tilde{P}\right) d \tilde{P} .
$$

The components of the flux $\mathbf{F}$ are then given by

$$
\begin{aligned}
F^{(\lambda)}= & u A+\frac{1}{2} \sigma_{0}\left(v_{e}^{2}-u_{e}^{2}\right) \cos \varphi-\frac{1}{2}(\mathrm{Fr})^{-1} \sigma^{2} \cos \varphi \\
& -\frac{\partial \chi}{\partial \varphi} \text { and } \\
F^{(\varphi)}= & v A-\sigma_{0} u_{e} v_{e} \cos \varphi+\frac{1}{\cos \varphi} \frac{\partial \chi}{\partial \lambda} .
\end{aligned}
$$

The mass function $m_{0}$, defined by

$$
m_{0}\left[P_{0}(\varphi)\right]=\int_{0}^{\varphi} \sigma_{0}(\tilde{\varphi}) \cos \tilde{\varphi} d \tilde{\varphi}
$$

is a measure of the mass of fluid contained between the $P_{0}$ contour and the equator, in the basic state. Note that we can now define $m_{e}=m_{0}(P)-m_{0}\left(P_{0}\right)$. The function

$$
\chi=\frac{1}{2} \sigma_{0} \psi_{e} u_{e} \cos \varphi
$$

is chosen so as to add a nondivergent "Plumb" correction to $\mathbf{F}$, which as described in $\mathrm{BH}$ and in WPP, results in the flux of stationary waves being independent of the phase of the waves, thereby making it much easier to interpret. The streamfunction of the nondivergent flow is $\psi$. The source-sink term can then be written in terms of the mechanical forcing $\mathbf{G}=(F, G)$ and thermal forcing $Q$ as

$$
\begin{array}{rr}
\mathcal{D}= & (\mathrm{Fr})^{-1} h_{\lambda} \sigma_{e} \\
& +Q_{0}\left(X-P_{e} m_{e}\right)+\left(\sigma\left[m_{e}-P_{e} m_{0}^{\prime}\left(P_{0}\right)\right]-\sigma_{e} m_{e}\right) \mathcal{F}_{0} \\
& (\text { Hadley cell dissipation }) \\
& +\left(X-P m_{e}-u_{e} \cos \varphi\right) Q_{e} \quad(\text { eddy heating term })
\end{array}
$$

\footnotetext{
${ }^{2}$ Note that we are using a different sign convention from $\mathrm{BH}$ for consistency with other pseudomomentum fluxes, for example, Plumb (1985).
}

$$
\begin{array}{r}
+m_{e}\left[G_{e \lambda}-(\cos \varphi)^{-1}\left(F_{e} \cos \varphi\right)_{\varphi}\right]-F_{e} \sigma_{e} \cos \varphi \\
\text { (eddy friction term) }
\end{array}
$$

where

$$
\mathcal{F}_{0}=-\frac{P_{0} Q_{0}}{\sigma_{0}}-\frac{\left(F_{0} \cos \varphi\right)_{\varphi}}{\sigma_{0} \cos \varphi}
$$

is the basic-state PV forcing. The terms in $\mathcal{D}$ are divided as above to facilitate the wave activity budgets presented in section 4. A clear distinction can be made between wave activity dissipation due to heating and friction acting directly on the eddies and that due to the forcing of the basic state. The latter is labeled as Hadley cell dissipation, as the forcing of the basic state is directly proportional to the strength of the Hadley circulation:

$$
F_{0}=\zeta_{a 0} v_{0} \text { and } Q_{0}=\frac{\left(v_{0} \sigma_{0} \cos \varphi\right)_{\varphi}}{\cos \varphi} .
$$

The Hadley cell dissipation contributes to $\mathcal{D}$ because of the nonconservation of wave activity $A$ in basic flows that are maintained by some external forcing (see, e.g., Haynes 1988). It provides an alternative possible sink for wave activity other than dissipation acting directly on the eddies. It is evident that the Hadley cell dissipation terms are zero in the no Hadley circulation experiments, which have been set up so that the eddy friction terms have the same form as in the Hadley circulation experiments. It should be noted that hyperdiffusion also enters the wave activity budget through $Q_{e}, F_{e}$, and $G_{e}$ in (14), and the hyperdiffusive dissipation of wave activity is treated as a separate term in the budgets of section 4 .

\section{Results from the numerical experiments}

\section{a. Zonally symmetric forcing}

Experiments were carried out with four different amplitudes of wave-3 forcing: $\epsilon=0.001$ (the linear case), $0.02,0.05$, and 0.2 . Figure 2 shows PV snapshots from the $\epsilon=0.05$ experiments. The Hadley circulation experiment is on the left, and the no-Hadley circulation experiment on the right. This figure shows three main differences between the two experiments.

1) In the Hadley circulation case the waves are observed to break in a region displaced $5^{\circ}-10^{\circ}$ north of the wave-breaking region in the no-Hadley circulation case.

2) A mean gradient of PV is present in the wave-breaking region of the Hadley circulation experiment, in every snapshot, whereas in the no-Hadley circulation case the PV has become largely homogenized throughout the breaking region by day 20 .

3 ) In the Hadley circulation case there appears to be a continuous cascade of enstrophy toward small scales (also noted in the experiments of HP), whereas once 

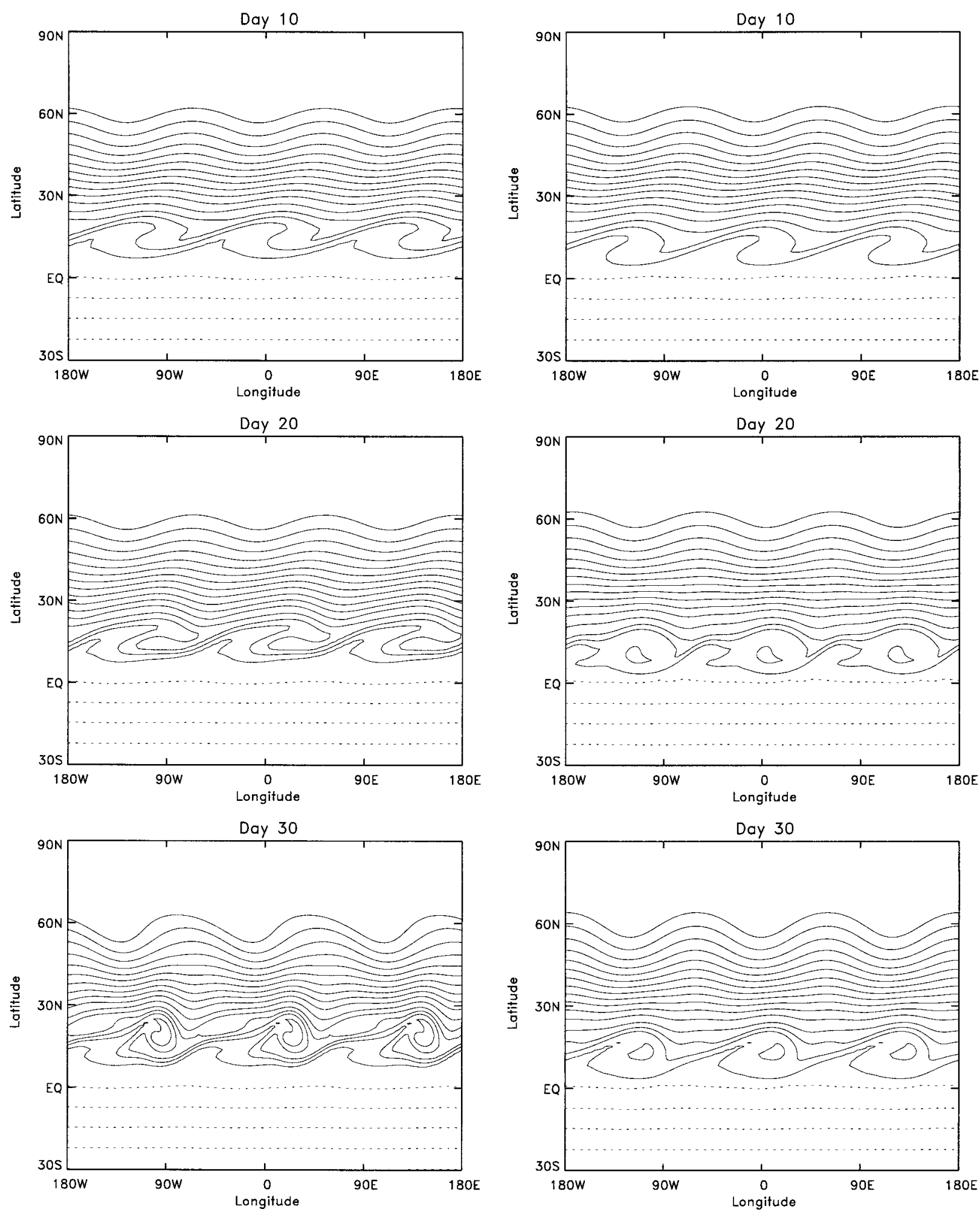

FIG. 2. Potential vorticity snapshots at days 10,20 , and 30 in the experiments with wave- 3 forcing at $\epsilon=0.05$. Left panels show the Hadley circulation case, and right panels the no-Hadley circulation case. Contour intervals are $0.15 \Omega \mathrm{H}^{-1}$, and negative contours are dotted. 
the PV has become well mixed in the critical layer region, the cascade slows dramatically when the Hadley circulation is absent.

It is also worth contrasting the lower two frames of Fig. 2: in the no Hadley circulation case the troughs and ridges of the waves become longitudinally aligned, suggesting the superposition of an incident and reflected wave train, whereas this does not happen in the Hadley circulation case.

Figure 3 shows the same snapshots for the $\epsilon=0.2$ experiments. These exhibit much the same differences as above. Greater wave amplitudes have led to a much broader wave-breaking region that becomes very well mixed when there is no Hadley circulation present. In the Hadley circulation case the wave-breaking region has an even broader extent, and there is continuous mixing of PV throughout it. A very strong gradient of PV develops in the extratropics by day 20 , and there is breaking to the north of this gradient by day 30. Further snapshots reveal sporadic wave-breaking events when high PV fluid is stretched and mixed into the low-latitude turbulent region. The Hadley circulation experiment exhibits much more turbulent, oscillatory behavior compared with the no Hadley circulation case, which settles into a small-amplitude oscillation, with weak wave breaking into the well-mixed region.

Figure 4 shows the evolution in latitude and time of the zonal mean meridional wave activity flux $\bar{F}^{(\varphi)}$ in each of the experiments. A natural definition of the absorptivity of a critical layer region is the jump in the meridional flux $\bar{F}^{(\varphi)}$ across the region. From the linear picture (upper panels), it is clear that the northward shift in the position of the breaking region, due to the Hadley circulation, is a linear phenomenon. Figure 5 shows the difference in $\boldsymbol{\nabla} \cdot \overline{\mathbf{F}}$ in the linear experiments, between the Hadley circulation case and the no Hadley circulation case. In the Hadley circulation case the maximum convergence of the flux is at $14.5^{\circ} \mathrm{N}$, where $\bar{u}=14 \mathrm{~m}$ $\mathrm{s}^{-1}$, and in the no Hadley circulation case it is at $9^{\circ} \mathrm{N}$, where $\bar{u}=1 \mathrm{~m} \mathrm{~s}^{-1}$. Away from these regions the momentum fluxes are nearly identical, indicating that the meridional wind has little effect on the linear propagation of the waves except where the zonal flow is weak. Held and Phillips (1990) discussed the linear propagation of barotropic waves on a sphere in the presence of a Hadley circulation. They noted the potential for some reflection at every latitude, due to latitudinal variations in the basic state, and the possibility of resonance due to reflection back from high latitudes. These experiments show that by day 11 , by which time the topography has been close to fully grown for a few days, the momentum fluxes in both experiments are approaching their final steady states. However, it takes until after day 20 before these final states are attained, suggesting the possibility of some weak linear reflection.

When $\epsilon=0.02$, Fig. 4 shows a clear qualitative effect of the presence of the Hadley circulation. When it is present (left panels), the flux is almost directly proportional to the linear flux, indicating near-complete absorption. When it is absent, the momentum fluxes first grow linearly, then decay away almost to zero, indicating that reflection is taking place from the critical layer region [see, e.g., the review in Haynes (1989)]. Note that the friction acting on the mean flow (4) is able to restore the PV gradients in the critical layer, allowing further absorption to take place. In the $\epsilon=$ 0.05 experiments the momentum fluxes in the Hadley circulation case remain reasonably close to the linear predictions, but with no Hadley circulation the reflection stage is reached more quickly (by day 18), and the critical layer is then observed to go through another cycle of absorption and reflection. At $\epsilon=0.2$ the presence of the Hadley circulation is not sufficient to absorb all of the incoming wave activity flux. In fact up until day 15 both experiments go through a similar-looking absorption-reflection cycle. After that, absorption is relatively weak in both experiments, but in the Hadley circulation case there are brief bursts of stronger absorption that correspond to wave-breaking events associated with the strong gradient of PV observed in the snapshots of Fig. 3.

\section{b. Isolated mountain forcing}

In the following set of experiments, the waves were forced by an isolated mountain situated at $45^{\circ} \mathrm{N}, 90^{\circ} \mathrm{W}$. Amplitudes of $\epsilon=0.001$ (linear case), 0.05, 0.2, and 0.5 were used. In the linear experiments (not shown), wave activity fluxes showed two wave trains generated by the mountain. One propagated northeastward and remained trapped at high latitudes by the layer of strong westerlies. The other much stronger wave train propagated southeastward and was absorbed at the linear critical line appropriate to each experiment, as in the wave-3 case.

Figure 6a shows snapshots of both PV (left panels) and wave activity density and flux (right panels) for days 8,16 , and 24 of the $\epsilon=0.05$ Hadley circulation experiment. In the extratropics the patterns are much as they are in the linear case. There is no evidence of any significant reflection from the critical layer region. However, there is a strong westerly flux of wave activity within the critical layer itself. This westerly flux of wave activity is most easily explained by advection due to the mean flow, as the waves now break in a region of significant westerly flow due to the northward shift in the position of the linear critical line. This effect greatly influences the wave activity budget in low latitudes for these experiments (see section $4 \mathrm{~b}$ ) and is one of the most substantial differences between the Hadley circulation and no Hadley circulation cases. Figure 6b shows the same pictures for the no Hadley circulation case. In this case there is strong evidence for a reflected wave train that is completely absent in the corresponding linear experiment. This wave train returns to mid- 

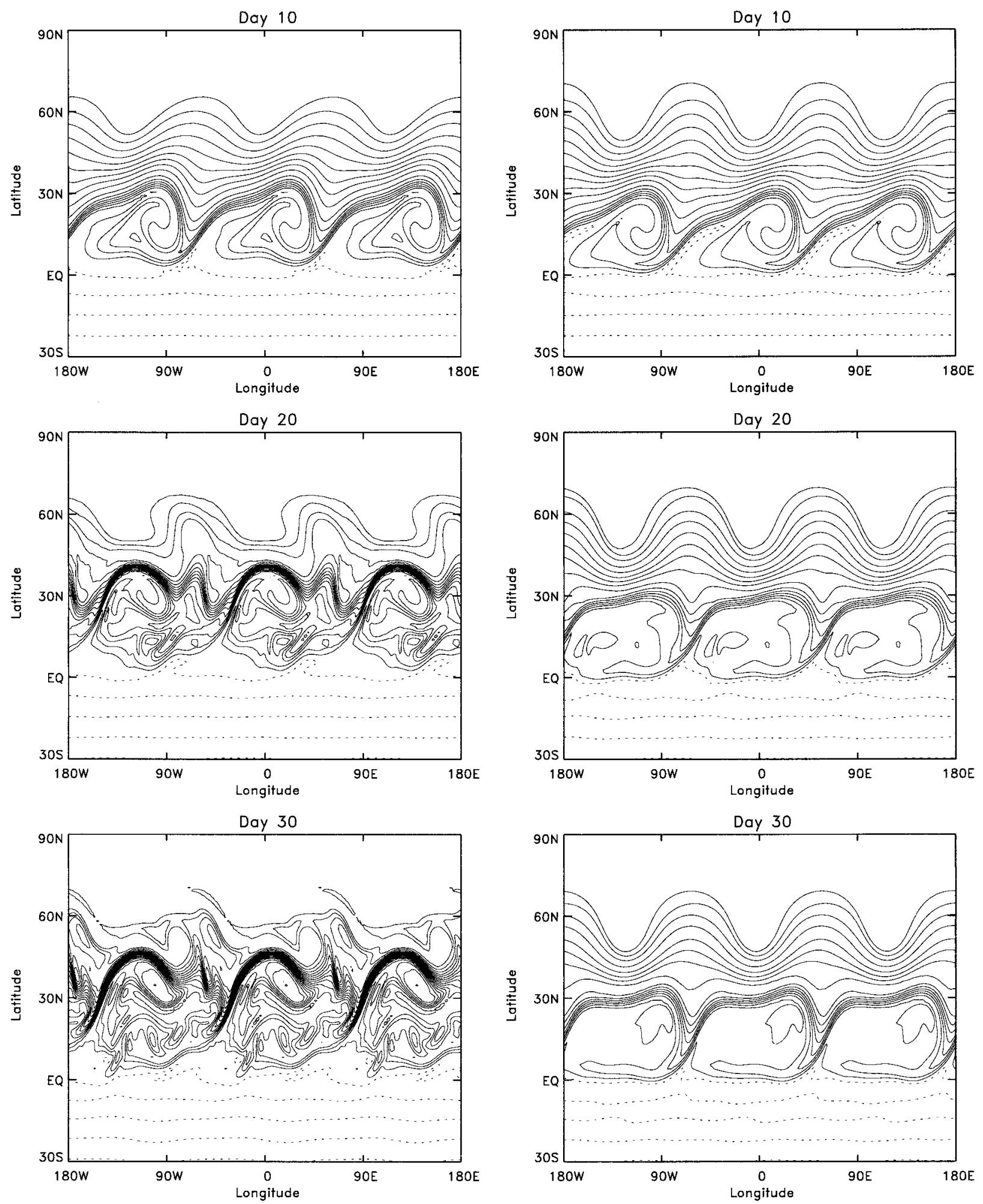

FIG. 3. As Fig. 2, but with $\epsilon=0.2$. 

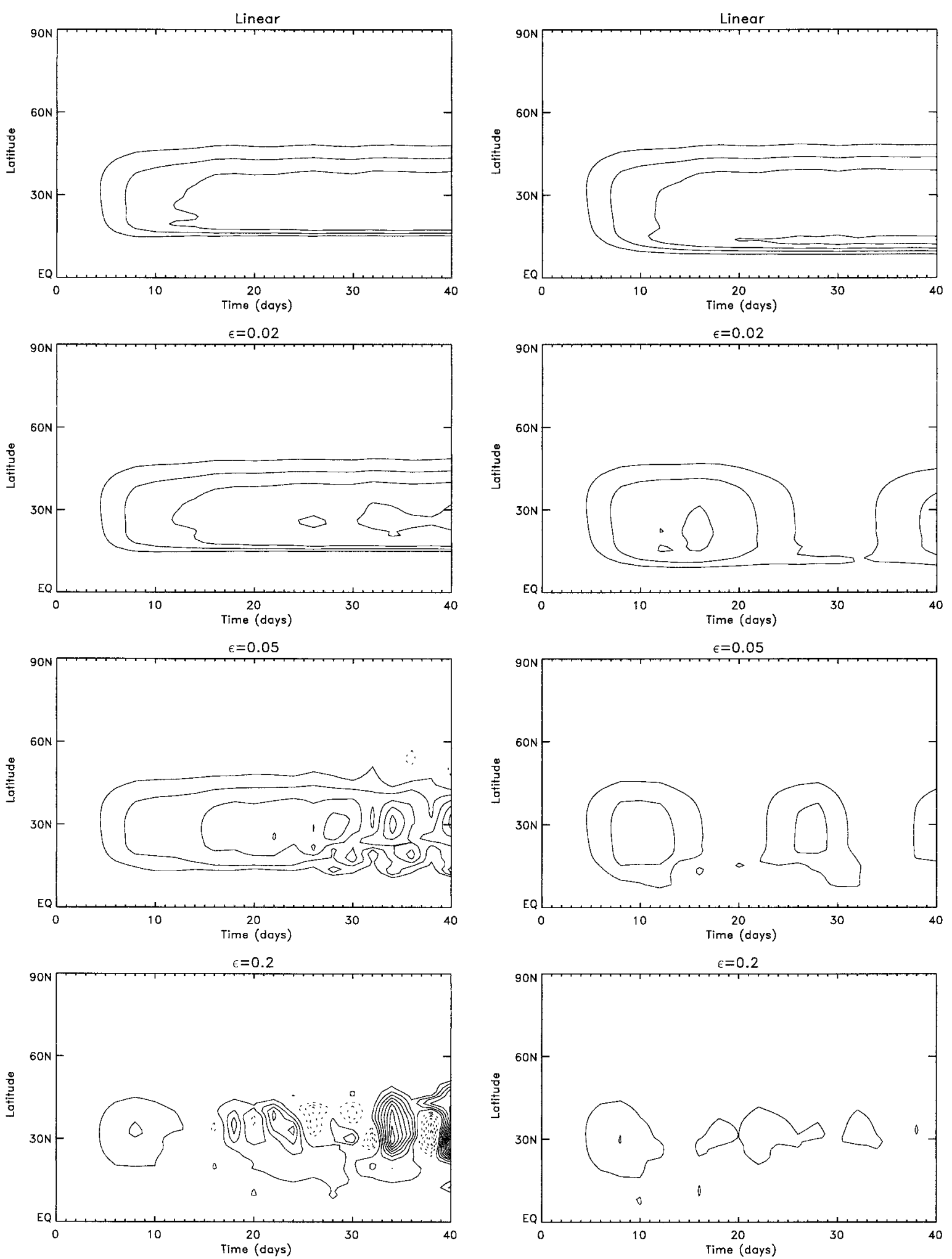

FIG. 4. Contour plot showing the evolution in latitude and time of the zonal mean meridional flux $\bar{F}^{(\varphi)}$ in the wave-3 experiments. Left panels show the Hadley circulation case, and right panels the no-Hadley circulation case. The cases shown are the linear case $(\epsilon \rightarrow 0), \epsilon$ $=0.02,0.05$, and 0.2. Contour intervals are $0.005 \epsilon^{2} \Omega^{2} a^{2} H$. Negative contours are solid and positive contours dotted, and the zero contour is omitted. 


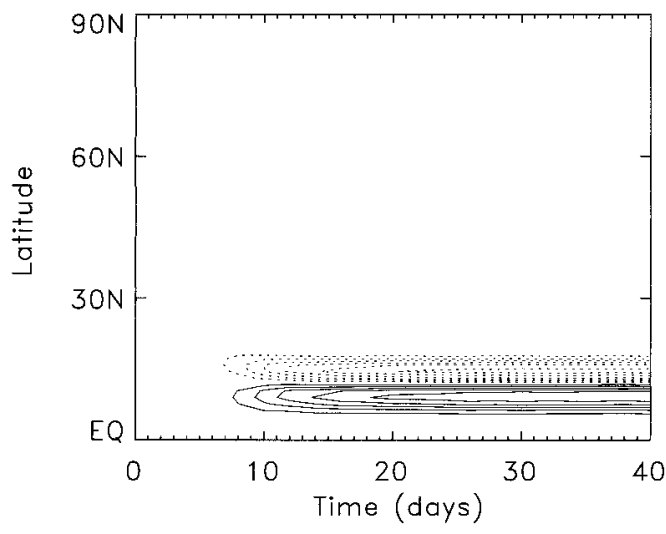

FIG. 5. Contour plot showing the difference in the evolution in latitude and time of $\boldsymbol{\nabla} \cdot \overline{\mathbf{F}}$ between the two linear experiments. The sense is $\langle$ Hadley circulation case $\rangle-\langle$ no-Hadley circulation case $\rangle$. The contour interval is $0.002 \epsilon^{2} H \Omega^{2} a$; positive contours are solid; and negative contours dotted. The zero contour is omitted.

latitudes where it is refracted back southward until it propagates back into the critical layer region around $90^{\circ}$ farther eastward. This is exactly the behavior reported in similar experiments by $\mathrm{BH}$, which were entirely inviscid except for hyperdiffusion. Unlike the Hadley circulation experiment, wave activity becomes concentrated at the longitude where the wave train reaches the critical layer, with relatively little downstream advection, instead of being stretched out across a broad region.

Figure 7 shows the evolution of the perturbation streamfunction $\psi_{e}$ in the $\epsilon=0.05$ experiments. The Hadley circulation case is on the left and the no Hadley circulation case is on the right. These snapshots are consistent with the wave activity snapshots of Fig. 6 and show a reflected wave train emerging from low latitudes in the no Hadley circulation case: this reflected wave train is entirely absent in the Hadley circulation case. (Note that the pictures are complicated slightly by the presence of the weaker wave train propagating northeast away from the mountain.) The reflected wave train propagates across midlatitudes before returning to low latitudes around $90^{\circ}$ eastward of the point of entry of the incident wave train.

Figures $8 \mathrm{a}$ and $8 \mathrm{~b}$ show snapshots from days 6, 12, and 18 of the $\epsilon=0.2$ experiments. Earlier times have been chosen as nonlinearity in both experiment sets is earlier due to the increased wave amplitude. Otherwise, the main effect of increasing the wave amplitude in the Hadley circulation case is to cause a weak reflected wave train to propagate out of the critical layer region. This wave train is noticeably stronger in the $\epsilon=0.5$ experiment (not shown), and this suggests that there is a limit to the magnitude of the incoming flux of wave activity that can be dissipated by the Hadley circulation or advected downstream before reflection must occur, as in the wave- 3 case. In the no Hadley circulation case the wave activity flux pictures are similar to the $\epsilon=0.05$ experiment, although the reflected wave train is somewhat stronger. However, it is worth remarking that by day 18 there is considerable mixing to the east of the initial breaking region noticeable in the PV plot. This appears to be due to the reflected wave train reentering the critical layer at a longitude that is distinct from where the original wave train enters.

\section{Discussion}

\section{a. Linear theory}

Schneider and Watterson (1984) and Farrell and Watterson (1985) discuss the problem of the southward propagation of linear stationary waves in both a shallow-water layer and on a quasigeostrophic $\beta$ plane. In the former paper the emphasis is on the possibility, when the mean meridional wind is to the south, of crossequatorial propagation across an easterly layer. The question of relevance here, the northward displacement of the critical line barrier to wave propagation when the mean meridional wind is to the north, is touched on by the latter paper. However, the fundamental question of where this barrier is actually located, in a general slowly varying flow, is not addressed. Here we revisit the quasigeostrophic $\beta$-plane analysis formulated in Schneider and Watterson (1984), with a view to answering this question. Assuming that the zonal mean wind $(\bar{u}, \bar{v})$ and quasigeostrophic PV gradient $Q_{y}$ vary slowly in $y$ (consistent with a WKB-type analysis), the appropriate perturbation equation is given by

$$
\left(\frac{\partial}{\partial t}+\bar{u} \frac{\partial}{\partial x}+\bar{v} \frac{\partial}{\partial y}\right) q^{\prime}+Q_{y} v^{\prime}=0,
$$

where $q^{\prime}=\nabla^{2} \psi^{\prime}-\psi^{\prime} / L_{R}^{2}$ is the perturbation quasigeostrophic PV, and $\psi^{\prime}$ is the streamfunction of the perturbation flow $\left(u^{\prime}, v^{\prime}\right)$. Note that here we are allowing the possibility of a finite Rossby radius $L_{R}=\sqrt{\left(g H / f^{2}\right)}$. The dispersion relation for linear waves $\psi^{\prime} \propto e^{i(k x+l y-\omega t)}$ is then given in terms of $\gamma=l / k$ by

$$
\begin{gathered}
\bar{v} \gamma^{3}+\bar{u} \gamma^{2}+x \bar{v} \gamma+x \bar{u}-Q_{y} / k^{2} \\
=0=\omega k^{-3}\left(k^{2}+l^{2}+L_{R}^{-2}\right),
\end{gathered}
$$

where $X=1+1 / k^{2} L_{R}^{2}$. (Note that $\omega$ may be set to zero without loss of generality, because in the case of nonzero $\omega, \bar{u}$ can be redefined to be the zonal velocity relative to the phase speed $\omega / k$.) As we are interested in the fate of southward propagating modes, $\gamma$ must also satisfy the group velocity condition

$$
\operatorname{Re} \frac{\partial \omega}{\partial l}(\gamma)=\bar{v}+\frac{2 \gamma}{\left(x+\gamma^{2}\right)^{2}} \frac{Q_{y}}{k^{2}}<0 .
$$

Since it is clearly not possible for any mode satisfying (18) to propagate past a line where $\operatorname{Re}(\partial \omega / \partial l)=0$ (cf. Farrell and Watterson 1985), this line becomes the location of the critical line barrier to linear propagation when $\bar{v} \neq 0$. 

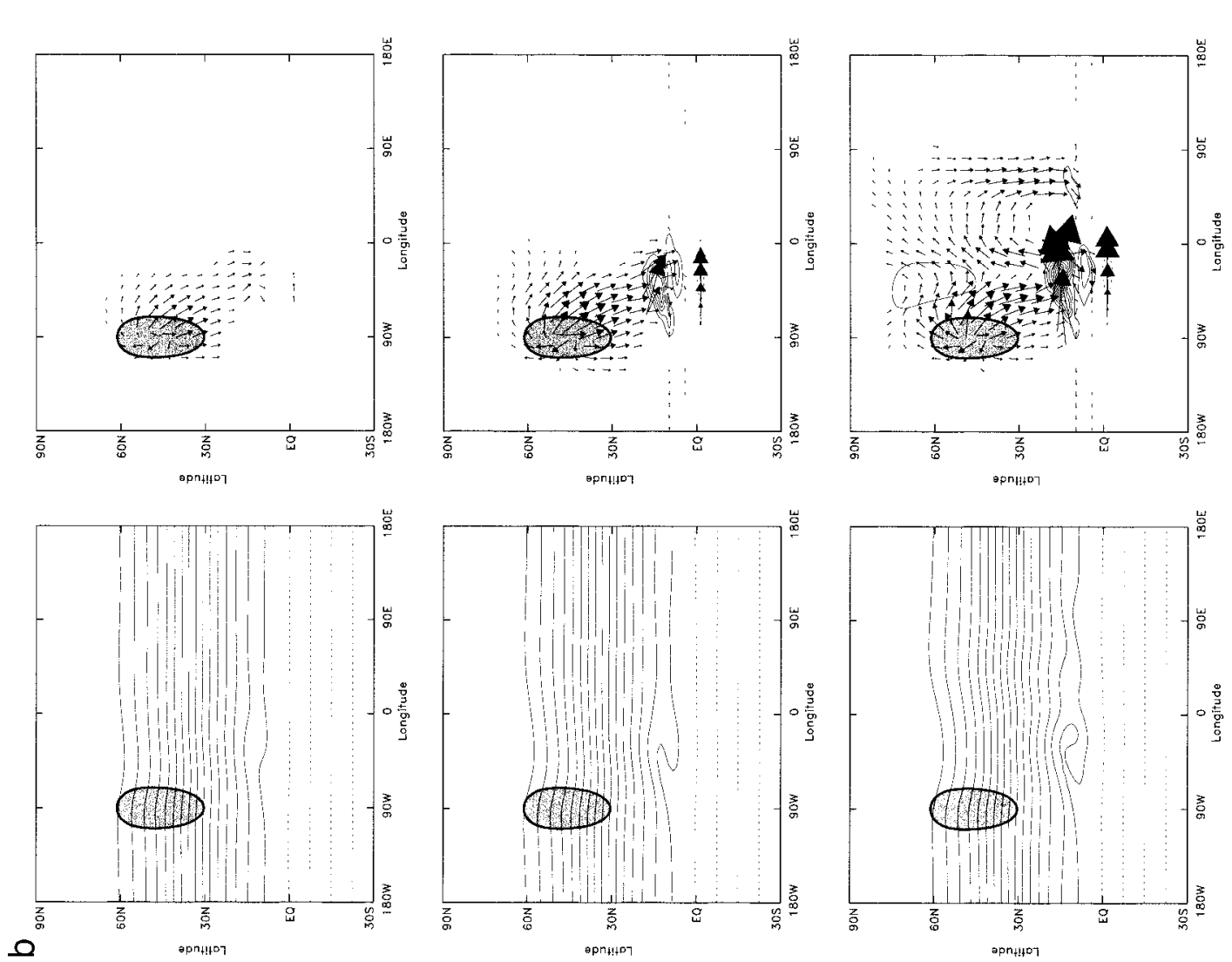

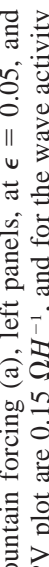
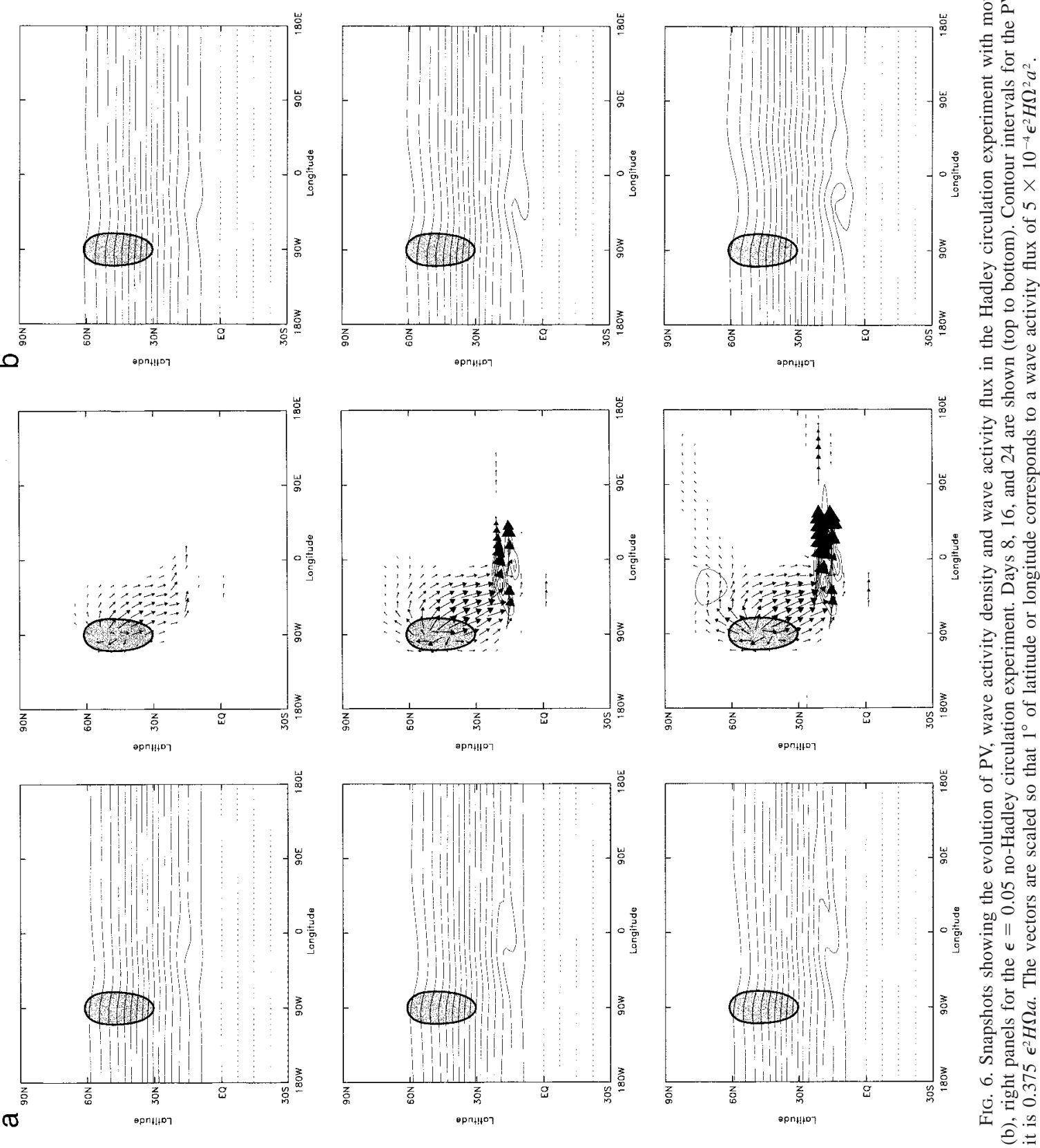

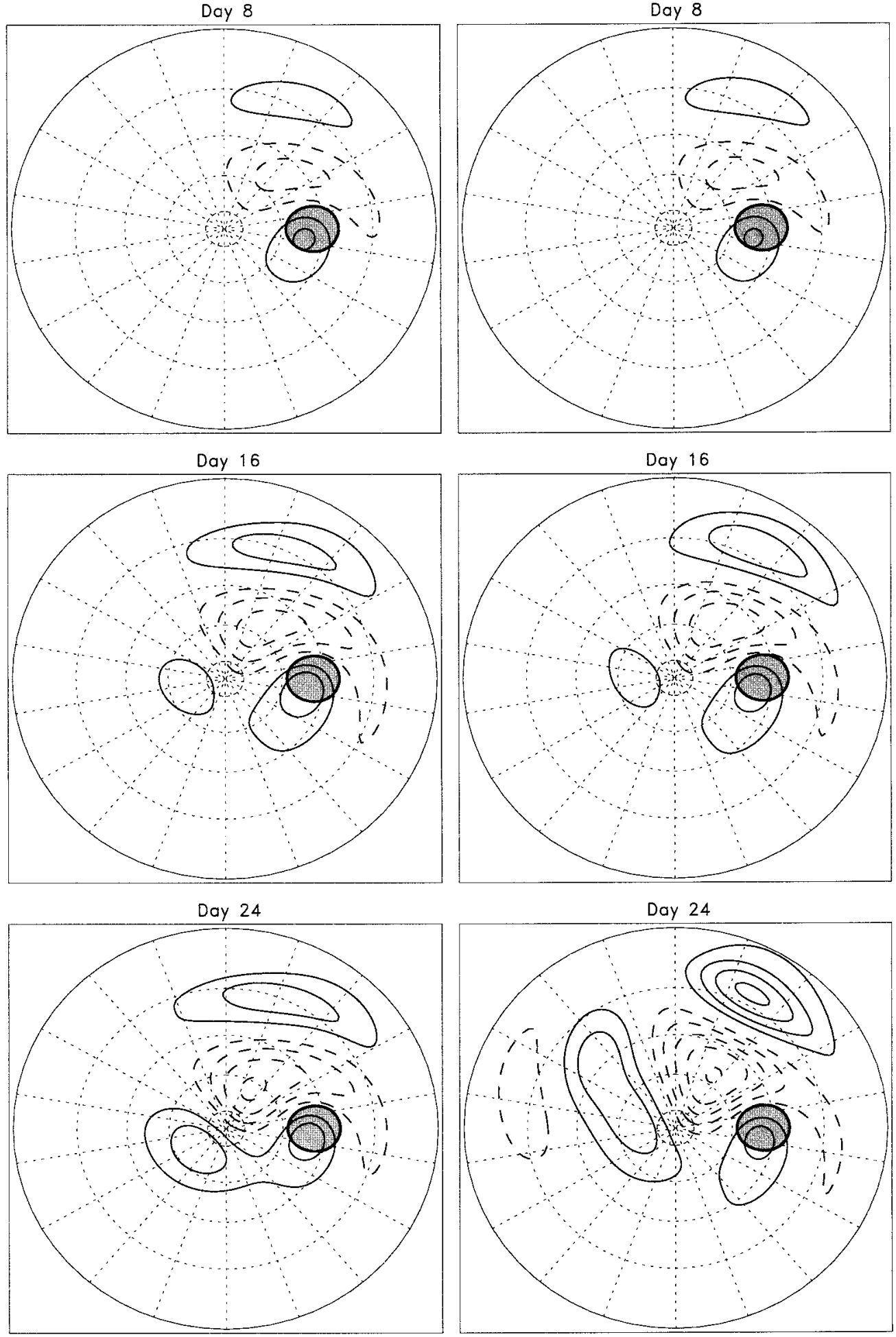

FIG. 7. Showing the evolution of the perturbation streamfunction $\psi_{e}$ in the $\epsilon=0.05$ Hadley circulation experiment (left panels) and no-Hadley circulation experiment (right panels). As in Fig. 6, days 8, 16, and 24 are shown (top to bottom). The contour interval is $2 \epsilon^{2} \Omega a^{2}$. Negative contours are dashed and the zero contour is omitted. 

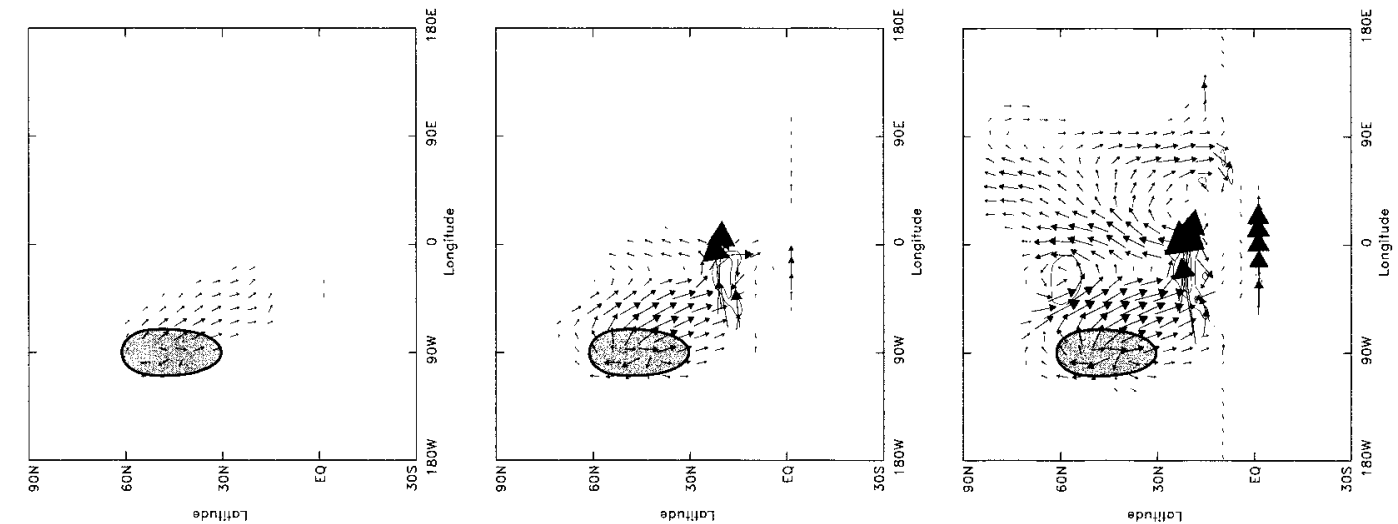

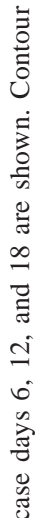
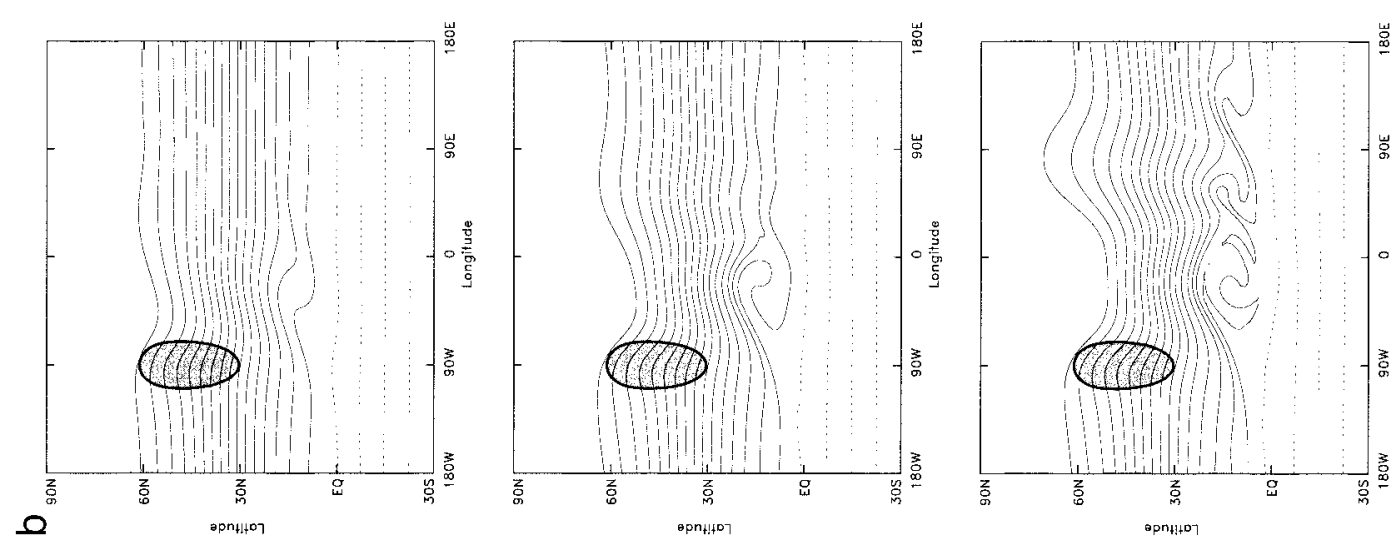

ำ
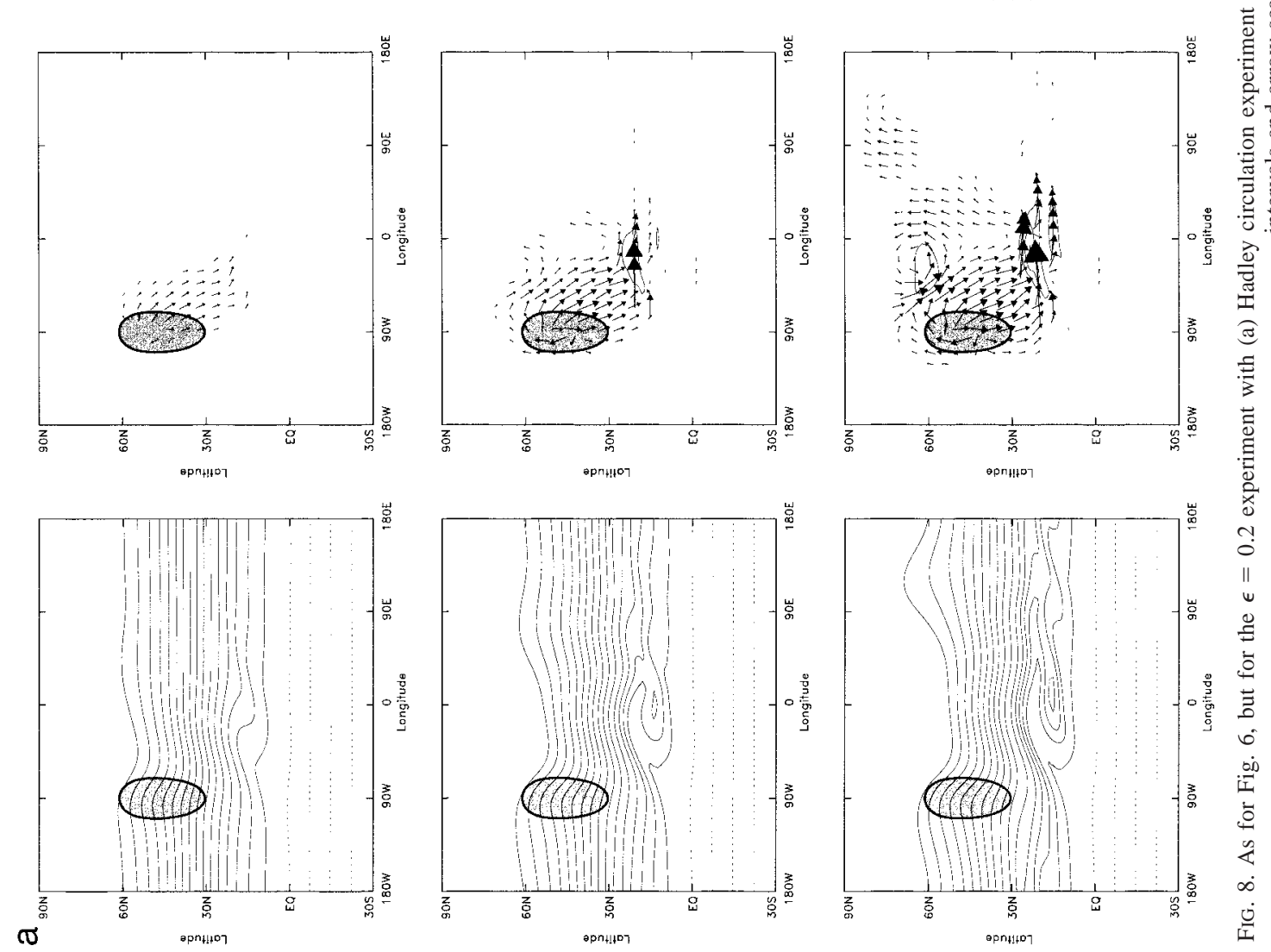

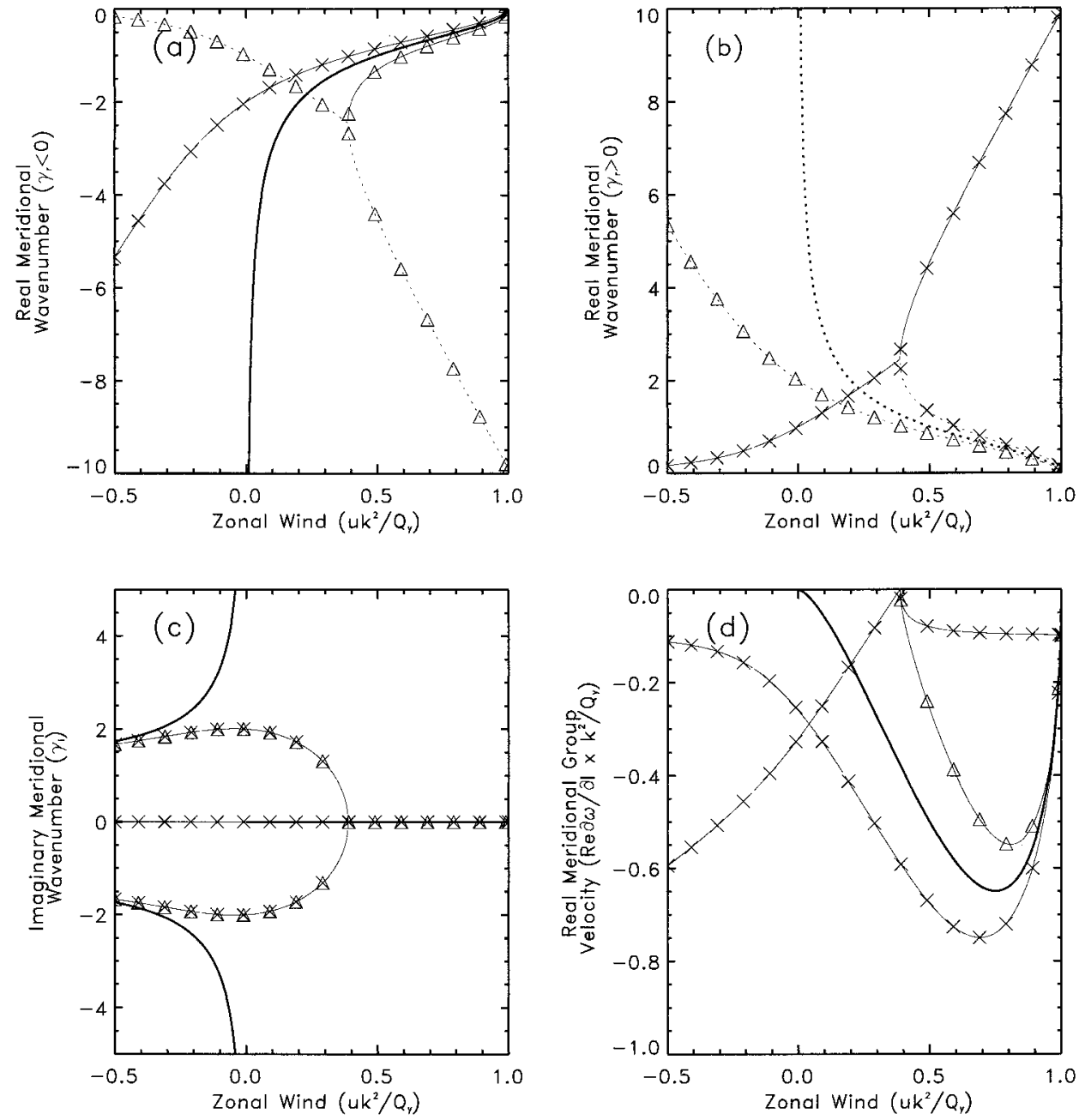

FIG. 9. Showing the dependence of the dispersion relation obtained for stationary waves in a $\beta$ channel, on the nondimensional zonal wind $\bar{u} k^{2} / Q_{v}$. The cases with meridional wind $\bar{v}<0$ (crosses), $\bar{v}=0$ (unmarked curves), and $\bar{v}>0$ (triangles) are shown. (a) Real meridional wavenumber $\gamma_{r}<0$. Northward propagating modes [defined as having $\operatorname{Re}(\partial \omega / \partial l)>0$ ] are plotted as dotted curves, and southward propagating modes as solid curves. (b) As in (a) but modes with $\gamma_{r}>0$. (c) Imaginary meridional wavenumber $\gamma_{i}$. (d) Real meridional group velocity $\operatorname{Re}(\partial \omega / \partial l)$. In (c) and (d) only southward propagating modes are shown. Further details are given in the text.

Figure 9 shows how the solutions to (18) change as the zonal wind is varied. Figures $9 \mathrm{a}$ and $9 \mathrm{~b}$ show $\gamma_{r}=$ $\operatorname{Re} \gamma$ for the three cases $\bar{v}=-0.1 Q_{\gamma} / k^{2}$ (crosses), $\bar{v}=$ 0 (thick curves), and $\bar{v}=0.1 Q_{y} / k^{2}$ (triangles). For simplicity, $L_{R}$ is taken to be infinite so that $X=1$. Figure 9a shows those branches with $\gamma_{r}<0$ and Fig. 9b those with $\gamma_{r}>0$. For the southward propagating modes, Fig. $9 \mathrm{c}$ shows the imaginary meridional wavenumbers $\gamma_{i}=$ $\operatorname{Im} \gamma$, and Fig. 9d shows the meridional group velocities. In Figs. 9a and 9b solid lines denote southward propagating modes, and for completeness, northward propagating modes are also included (dotted lines), though these will not be discussed in what follows. (A suitable dimensionalization for the shallow-water model of section 3, at low latitudes, would be to take $Q_{v} / k^{2}=50 \mathrm{~m}$ $\mathrm{s}^{-1}$, appropriate for zonal wavenumber 3 , which yields $\bar{v}= \pm 5 \mathrm{~m} \mathrm{~s}^{-1}$. Then $\bar{u}$ varies between -25 and $50 \mathrm{~m}$ $\mathrm{s}^{-1}$ along the $x$ axis.)

Consider first the case $\bar{v}=0$, when the Hadley circulation is absent. The unmarked thick solid curve in Fig. 9a shows that a propagating mode exists for $0<$ $\bar{u}<Q_{v} / k^{2}$. This is the well-known Charney-Drazin criterion for this particular model. The corresponding $\gamma_{i}-$ solid curves in Fig. 9c-show that the mode becomes purely evanescent for $\bar{u}<0$. For $\bar{u}>Q_{v} / k^{2}$ the zonal winds are too strong for stationary Rossby wave propagation, and again only evanescent waves are possible. This upper limit on $\bar{u}$ is very insensitive to the presence of a meridional current.

In the case of southerly mean meridional wind $\bar{v}>$ 0 , the range of zonal winds that allow a southward propagating mode is clearly reduced (triangles in Fig. 9a). 
Propagation is no longer possible when $\bar{u}$ is less than a critical value $\bar{u}_{c}$, which for the plotted case $(\bar{v}=$ $\left.0.1 Q_{y} / k^{2}\right)$ is approximately equal to $0.38 Q_{y} / k^{2}$. For $\bar{u}<$ $\bar{u}_{c}$ the real group velocity (not shown) is northerly, and the mode splits into two complex conjugate mixed propagating-evanescent solutions. The two possible values of $\gamma_{i}$ are illustrated in Fig. 9c.

To derive an approximate expression for $\bar{u}_{c}$, we proceed as follows. Figure 9d shows that the group velocity of the propagating mode goes to zero at $\bar{u}_{c}$, and in Fig. $9 \mathrm{c}$ it is clear that the mode also splits into two mixed propagating-evanescent branches for lower values of $\bar{u}$. One can see that the meridional group velocity is always zero at this branch point, where $\gamma=\gamma_{0}$, as the dispersion relation there must have the form

$$
\omega=\mathcal{F}(k, \gamma)\left(\gamma-\gamma_{0}\right)^{2}\left(\gamma-\gamma_{1}\right)
$$

and therefore $\partial \omega / \partial l\left(\gamma_{0}\right)=k^{-1} \partial \omega / \partial \gamma\left(\gamma_{0}\right)=0$, provided that the function $\mathcal{F}$ is well behaved at $\gamma=\gamma_{0}$, which it is for all possible coefficients. (The $\gamma_{1}$ in this expression is the other root of (18), which is on the northward propagating branch.) This means that the condition for the existence of three real roots of (18) is also the condition for the existence of a southward propagating mode. This condition is [from the basic theory of cubic polynomials, e.g., Abramowitz and Stegun (1972)]

$$
\begin{aligned}
& \frac{X}{27}\left(\frac{\bar{u}}{\bar{v}}\right)^{4}-\frac{1}{27} \frac{Q_{y}}{\bar{v} k^{2}}\left(\frac{\bar{u}}{\bar{v}}\right)^{3}+\frac{2 X^{2}}{27}\left(\frac{\bar{u}}{\bar{v}}\right)^{2}-\frac{X}{3} \frac{Q_{y}}{\bar{v} k^{2}} \frac{\bar{u}}{\bar{v}} \\
& \quad+\frac{1}{4}\left(\frac{Q_{y}}{\bar{v} k^{2}}\right)^{2}+\frac{X^{3}}{27}<0 .
\end{aligned}
$$

In the limit $\bar{u}, \bar{v} \ll Q_{y} / k^{2}$, this gives the location of the barrier to propagation at

$$
\bar{u}_{c} \approx\left(\frac{27}{4}\right)^{1 / 3}\left(\frac{Q_{y}}{k^{2}}\right)^{1 / 3} \bar{v}^{2 / 3} .
$$

Note that this expression is independent of $x$ and, thus, of the Rossby radius.

For the numerical experiments with the shallow-water model in the linear regime $(\epsilon=0.001)$, using $\bar{v}=1.5$ $\mathrm{m} \mathrm{s}^{-1}$ from Fig. 1, and $Q_{y} / k^{2}=50 \mathrm{~m} \mathrm{~s}^{-1}$, expression (22) yields $\bar{u} \approx 10 \mathrm{~m} \mathrm{~s}^{-1}$. This is in reasonable agreement with the location of maximum convergence of $\mathbf{F}$ at $\bar{u}=14 \mathrm{~m} \mathrm{~s}^{-1}$ (see Fig. 5 and the accompanying text).

Finally, in the northerly wind case $\bar{v}<0$ (crosses in Fig. 9a), the main result of Schneider and Watterson (1984) is easily seen, namely, that southward propagation is possible through easterlies in this case. However, in strong easterlies, $|\gamma|$ becomes large, indicating that finite-amplitude waves would be likely to break in practice. A second southward propagating mode, with positive $\gamma_{r}$ (crosses in Fig. 9b), exists when $\bar{u}$ is greater than $\bar{u}_{c}^{*}$, where $\bar{u}_{c}^{*}$ is equal to $\bar{u}_{c}$ calculated for the same magnitude of southerly winds. This mode has meridional group velocity close to $\bar{v}$, as illustrated by the crosses on the top right curve in Fig. 9d for which $\bar{v}$
$=-0.1 Q_{y} / k^{2}$. For $\bar{u}<\bar{u}_{c}^{*}$ this second mode becomes mixed propagating-evanescent and (from Fig. 9c) has the same possible imaginary wavenumbers as the mixed mode in the corresponding southerly wind case.

\section{b. Nonlinear behavior}

In order to gain a deeper understanding of the differences between the individual pairs of experiments, a wave activity budget was calculated for both the $\epsilon=$ 0.05 wave- 3 and the $\epsilon=0.2$ isolated mountain sets of experiments. These two sets of experiments are chosen as the total wave activity in each is comparable. In the wave- 3 case the budget was taken for the region south of $25^{\circ} \mathrm{N}$. From the expression for $\mathcal{D}$ in the wave activity relation (14), a distinction can be made between the different ways in which the wave activity is dissipated. The budget is therefore divided up into the incoming nonadvective part of the meridional component of the wave activity flux, the incoming advective part of the flux, dissipation due to the forcing acting on the basic state (hereafter Hadley cell dissipation), dissipation due to friction acting on the eddies, dissipation due to heating acting on the eddies, and dissipation due to hyperdiffusion. Figure 10a shows the results for the $\epsilon=0.05$ Hadley circulation case. The incoming nonadvective component of the wave activity flux (the light solid curve) increases over the first 10-20 days to a maximum that is fairly close to the linear prediction of $4.79 \times$ $10^{-5} H \Omega^{2} a^{2}$ (calculated by scaling the appropriate flux from the linear experiment). The remaining curves show that about $50 \%$ of this incoming wave activity is dissipated by the hyperdiffusion term, with the remainder either advected back out of the region, dissipated by friction directly acting on the eddies, or by the Hadley cell dissipation. Only the direct effect of heating on the eddies was found to be unimportant in the budget. It was confirmed that the sum of all the tendencies (thick solid curve) was in close agreement with the rate of change of total wave activity in the region.

Figure 10b shows the same budget for the experiment without the Hadley circulation. In this experiment the total wave activity in the critical layer oscillates with a period of around 15 days. It is clear from the budget that this oscillation is entirely due to variations in the nonadvective component of the incoming flux, which can also been seen in Fig. 4. These variations in the incoming flux are consistent with periods of nonlinear reflection from the critical layer, and these result in the incoming flux falling far short of the linear prediction of $4.75 \times 10^{-5} H \Omega^{2} a^{2}$. Dissipation is fairly constant and is due to the direct effect of friction on the eddies, and to hyperdiffusion, although the latter term is much weaker than in the Hadley circulation case. It appears that the rate of total dissipation is too slow to prevent the critical layer from becoming "saturated" with wave activity, thereby forcing reflection to take place, as must happen in the case of the simple, inviscid, barotropic 

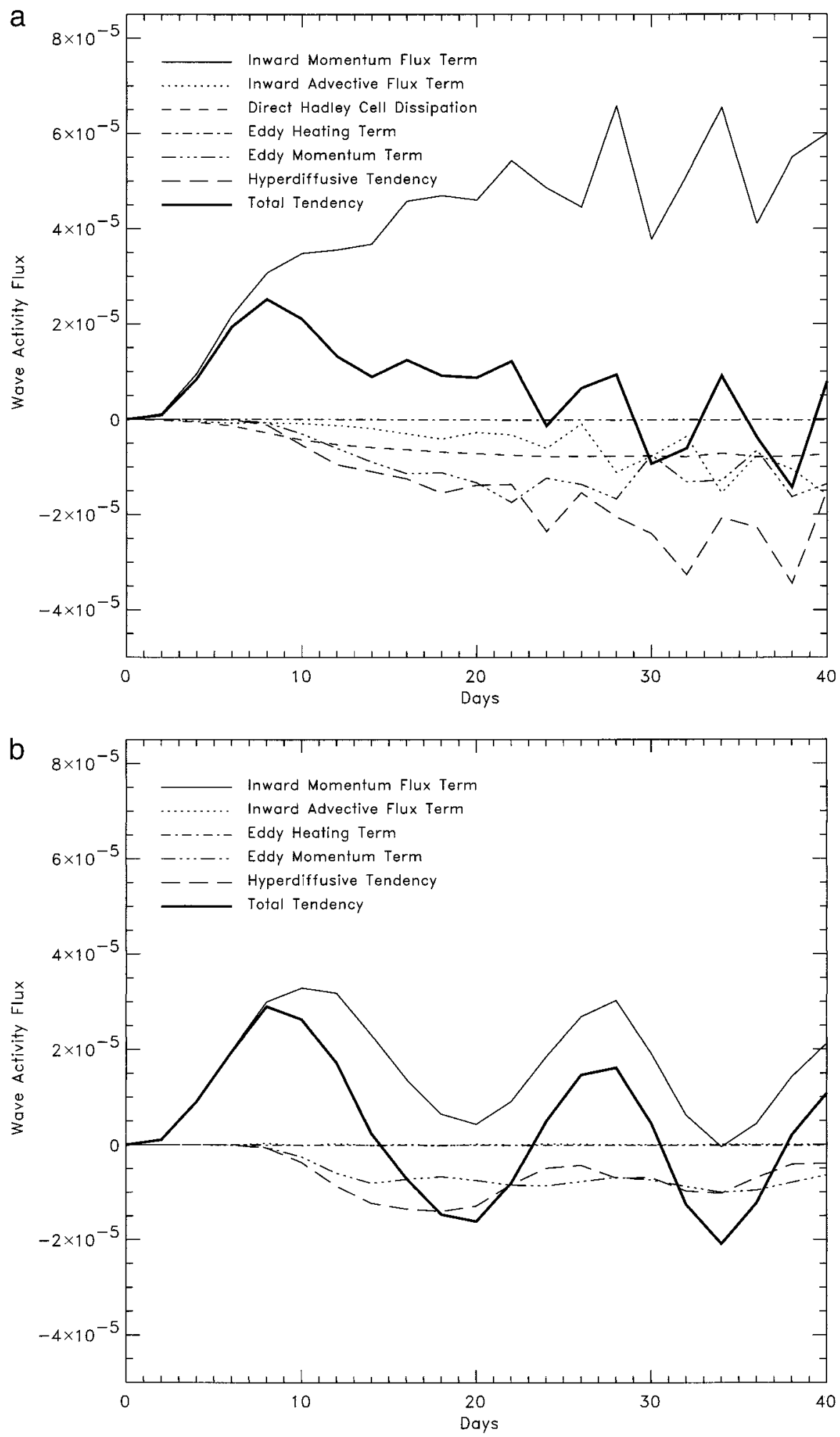

FIG. 10. Graph showing the evolution in time of various terms in the wave activity budget for the region south of $25^{\circ} \mathrm{N}$ in the wave-3 Hadley circulation experiment with $\epsilon=0.05$. Units are $H \Omega^{2} a^{2}$. (b) As (a), but for the equivalent no-Hadley circulation experiment. 

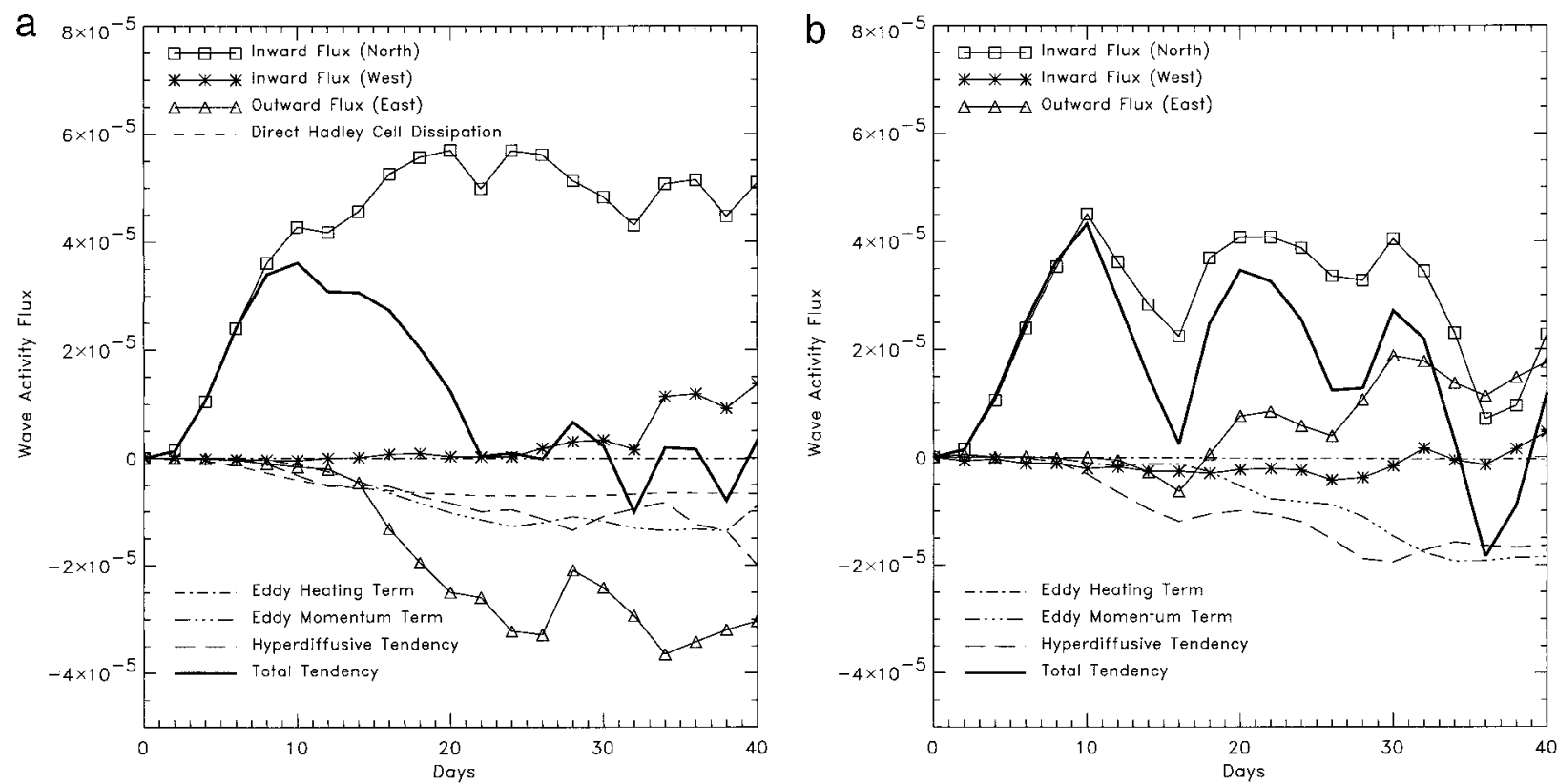

Fig. 11. As Fig. 10a, but for the isolated mountain Hadley circulation experiment with $\epsilon=0.2$. The budget in this case is for a region bounded to the north by the $25^{\circ} \mathrm{N}$ latitude circle and to the west and east by the $135^{\circ} \mathrm{W}$ and $45^{\circ} \mathrm{E}$ meridians, respectively. (b) As (a), but for the corresponding no-Hadley circulation experiment.

critical layer discussed by Killworth and McIntyre (1985, section 6). By contrast, in the Hadley circulation case the rate of dissipation is sufficient to prevent such saturation. Perhaps surprisingly, most of this extra dissipation is achieved not through the direct effect of the Hadley cell forcing acting on the basic state, but instead it is due to the more vigorous cascade of enstrophy to small scales that leads to greater dissipation by the hyperdiffusion term. The proof in Killworth and McIntyre (1985, section 7) states that if dissipation terms act only as a flux of PV within a barotropic critical layer and do not diffuse vorticity into it, then the time-integrated absorptivity of the critical layer will remain bounded. The hyperdiffusion terms taken alone come close in practice to satisfying this condition in the shallow-water experiments. $^{3}$ This suggests that the unbounded time-integrated absorptivity due to the hyperdiffusion terms in our experiments is entirely a consequence of the presence of the Hadley cell forcing and the eddy friction.

Figures $11 \mathrm{a}$ and $11 \mathrm{~b}$ show the budgets for the $\epsilon=$ 0.2 isolated mountain experiments, for the Hadley circulation and no-Hadley circulation cases, respectively. In this case the budgets are for a region bounded to the north by the $25^{\circ} \mathrm{N}$ latitude circle and to the west and east by the $135^{\circ} \mathrm{W}$ and $45^{\circ} \mathrm{E}$ meridians, respectively. This allows us to assess the relative importance of down-

\footnotetext{
${ }^{3}$ Although it should be noted for this argument to strictly apply it must be assumed that under the conditions of balanced flow discussed in $\mathrm{BH}$, that the Killworth and McIntyre proof applies to the shallowwater system that we are considering.
}

stream radiation of wave activity in the budgets of the two experiments. ${ }^{4}$ In the Hadley circulation experiment, after day $20,50 \%-60 \%$ of the incoming wave activity flux is radiated away at the eastern boundary, making it by far the most important term in the budget. The eastern boundary flux was found to be dominated by the advective component, with the nonadvective component in the westward direction and equal to about $40 \%$ of the magnitude of the advective component. In the no-Hadley circulation experiment the total wave activity in the region was found to increase to almost double that in the Hadley circulation case, despite a weaker incoming flux at the northern boundary due to reflection. At the eastern boundary there is no westerly flux due to downstream advection; in fact, there is an easterly flux as the reflected wave train is refracted around, and enters, the region from the east (this can be seen in the day-18 panel of Fig. 8b).

In conclusion, therefore, the main effect of the Hadley circulation in the wave- 3 case is the increase in the absorption of wave activity, primarily by increased dissipation due to an increase in the strength of the enstrophy cascade. In the isolated mountain case this increased dissipation, while still significant, is swamped by the larger effect of the downstream advection of wave activity in the Hadley circulation case, which can be

\footnotetext{
${ }^{4}$ Note that in this budget we are showing the sum of the advective and nonadvective components of the meridional flux, rather than showing them separately as in Fig. 10.
} 
explained by the waves breaking in a region of westerly winds.

\section{Long-time behavior}

The effect of the Hadley circulation on the longtime equilibrium behavior of this simple model is also of interest. Recently, Polvani et al. (1999) showed that, under certain conditions, a shallow-water flow without a Hadley circulation, in which waves are forced by an isolated mountain, can exhibit spontaneous low-frequency variability. If the mountain size is sufficiently large, then this variability typically involves repeated vortex shedding from the northern flank of the midlatitude jet, at some distance downstream of the mountain. The structure of the streamfunction anomaly associated with this vortex shedding is that of a wave train eminating from the Tropics. This wave train is qualitatively similar in structure to both atmospheric teleconnection patterns such as the PNA pattern (see, e.g., Frederiksen and Webster 1988) as well as the most unstable normal modes calculated when the equations of motion are linearized about the 300-mb time mean flow (see, e.g., Simmons et al. 1983).

The effect of the Hadley circulation on this type of oscillation was therefore investigated by integrating the $\epsilon=0.5$ isolated mountain experiments for 800 days and then analyzing the final 100 days of these integrations. For these long runs, the location of the top of the mountain was moved southward to $36^{\circ} \mathrm{N}$, in order to cause the maximum possible interaction between the jet and the mountain. It was also found to be necessary to increase the magnitude of the hyperdiffusivity to $1 \times$ $10^{-11} a^{6} \Omega$, in order to maintain numerical stability. Otherwise, the experiments were identical to those described in sections 2-4.

In both experiments the flow remains unsteady throughout the period of integration. Figure 12 shows the time mean PV, averaged over the final 100 days, in both the Hadley circulation and the no-Hadley circulation cases. The main differences between these plots are that in the Hadley circulation experiment the PV north of $60^{\circ} \mathrm{N}$ is relatively well mixed, and south of $30^{\circ} \mathrm{N}$ in the critical layer region, wave breaking appears to be a much stronger ongoing process, especially between $90^{\circ} \mathrm{W}$ and $130^{\circ} \mathrm{E}$. Both of these differences are consistent with those seen in the PV snapshots of the initial-value wave-3 experiments (Figs. 2 and 3).

Figure 13 shows the leading complex empirical orthogonal functions (EOFs) of the perturbation streamfunction field $\psi^{e}$ (defined as the deviation from the time mean) as well as their complex principal components. The reader is referred to Polvani et al. (1999) for a description of how this method of analyzing the leading order variability of the streamfunction field is applied in these experiments, and to Horel (1984) for a more general review of complex EOFs. Figure 13a shows the Hadley circulation case. The leading order variability
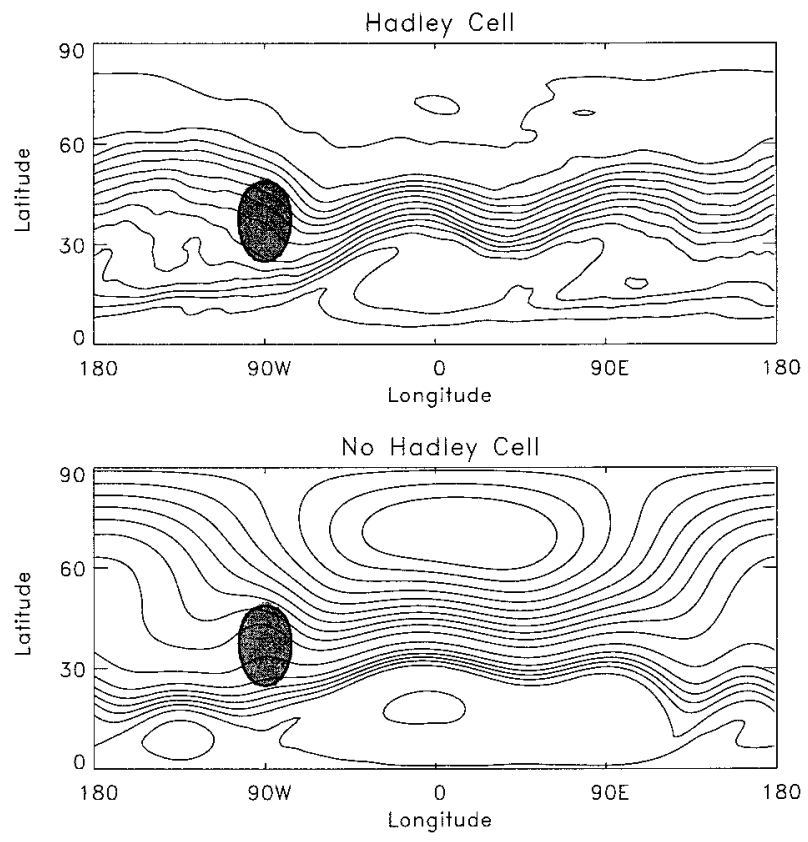

FIG. 12. Contour plots showing the time mean PV fields in the longtime $\epsilon=0.5$ isolated mountain experiments. The contour intervals are $0.2 \Omega H^{-1}$.

has the structure of a zonal wavenumber-4 wave train, which is fairly constant in amplitude around the globe, except for a minimum in the strong jet region just downstream of the mountain. The principal components indicate that the phase propagation is to the east and the period is around 10 days. The PV snapshots (not shown) show large waves in the tight midlatitude PV gradient that extend around the globe. This is in sharp contrast to Fig. 13b, showing the no Hadley circulation case, where a wave train extends from the Tropics at the opposite side of the globe, across the Pole to the mountain. In this case the phase propagation is out of the Tropics toward the mountain, and the period appears to be approximately 80 days. This type of wave train is very similar to that in several experiments reported in Polvani et al. The PV snapshots (not shown) show a small vortex being shed from the northern flank of the jet over this time. The total amplitude of this oscillation is substantially smaller than in the Hadley circulation case.

In the zonally symmetric wave- 3 experiments of HP, the flow settles into a periodic oscillation above a certain forcing amplitude. They describe this oscillation as being due to an instability associated with the wave breaking. It seems likely that the strength of such an instability is proportional to the strength of the wave breaking itself, which in the longtime limit has been shown to be much stronger when the Hadley circulation is present. This is a possible explanation for the much greater longtime variability present in the Hadley circulation experiment, and this brief study seems to motivate a detailed investigation into this effect in a more realistic model. 

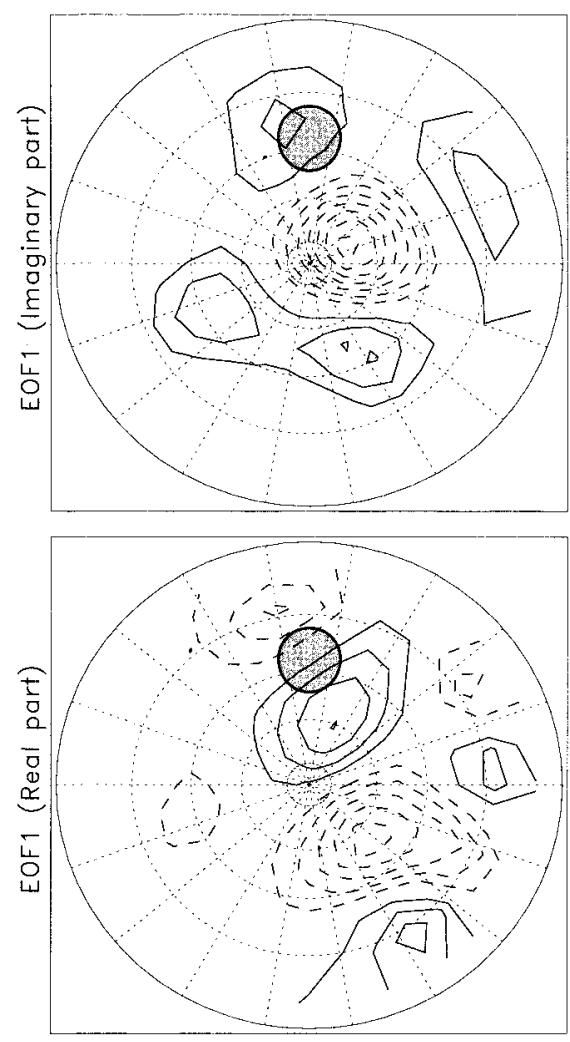

요

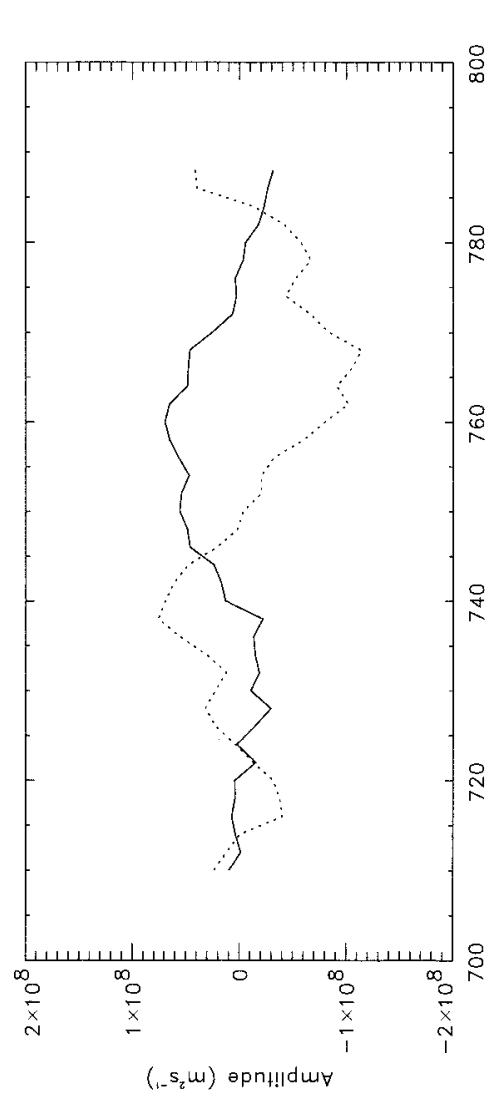

흘을

I

苛

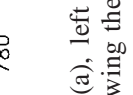
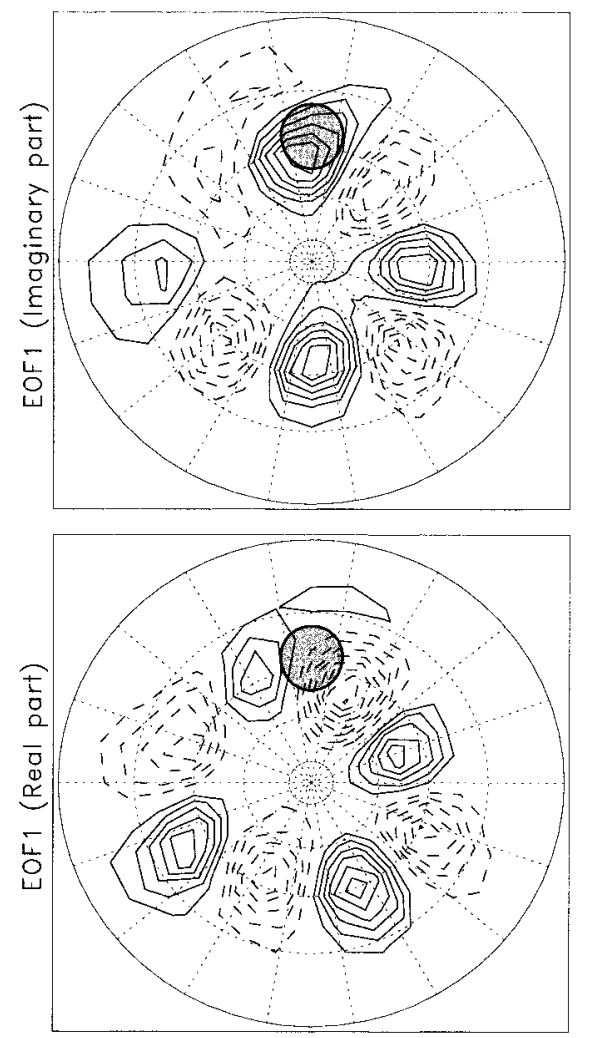

$\sigma$

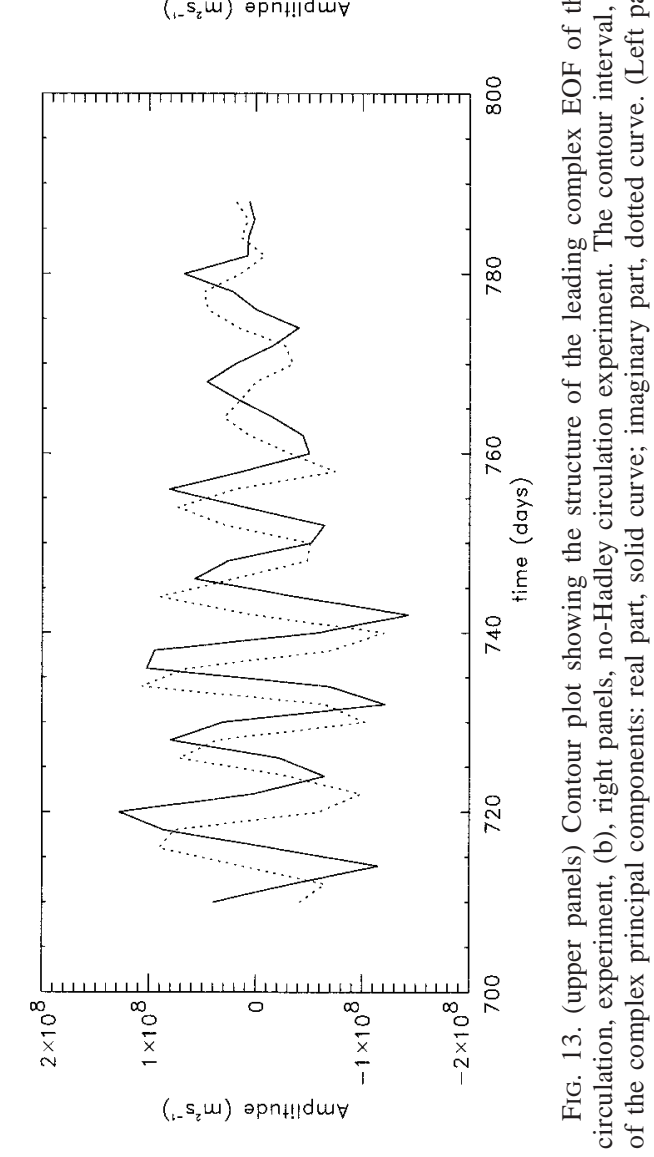




\section{Summary and conclusions}

In this paper we have addressed the question of how a representation of the Hadley circulation affects the propagation and nonlinear reflection of planetary waves in the winter hemisphere of a shallow-water layer. This was achieved by comparing results from numerical experiments with initial flows that contained a forced Hadley circulation, with those from initial flows that were similar in every other detail but with no Hadley circulation.

Firstly, the absorption-reflection characteristics of the two flows were compared by forcing waves in the midlatitudes with a zonal wave-3 topography. In the linear limit, the presence of the Hadley circulation is found to displace the critical line where the waves are absorbed poleward, an effect that has been previously discussed by Schneider and Watterson (1984) and Farrell and Watterson (1985). At finite forcing amplitudes the waves are found to break in critical layer regions around their respective critical lines. The Hadley circulation flows remain in a regime of near-complete absorption of wave activity at the same forcing amplitudes at which the no Hadley circulation flows exhibited substantial nonlinear reflection. At high forcing amplitudes, the Hadley circulation flows would also reflect wave activity, but less efficiently than the corresponding no Hadley circulation flows. An analysis of the wave activity budgets in the critical layer regions shows that in the Hadley circulation flows, absorption is maintained through increased dissipation of wave activity in the wave-breaking region. This increased dissipation occurred principally due to an increased cascade of enstrophy to small scales, consistent with more sustained wave breaking. The wave breaking is sustained by the continual restoration of the basic-state PV gradient in the wave-breaking region due to the presence of the Hadley circulation, and this prevents the critical layer region from becoming well mixed and, hence, from becoming an efficient reflector.

The behavior of wave trains generated in the extratropics by an isolated mountain was then investigated. In the absence of the Hadley circulation, and at sufficient forcing amplitudes, zonally localized wave breaking led to the wave train being reflected at the same longitude at which it entered the critical layer region. Similar experiments showing localized nonlinear reflection have been previously reported by BH and WPP. In the Hadley circulation case the behavior is quite different. Not only was there increased dissipation of wave activity in the critical layer region, as in the wave- 3 experiments, but there was also a considerable westerly flux of wave activity within the critical layer. Further analysis has shown that this flux is largely advective and, therefore, is largely due to the fact that the waves tend to break in relatively strong westerly flow when the Hadley circulation is present. Wave activity budgets of a longitudinally localized region show that the westerly flux is the main contributor to the enhanced absorptivity, at least before the waves have propagated around the globe and entered the region from the west. The importance of the location of the critical line to the wave activity budget motivated the analysis of section $4 \mathrm{a}$ where we obtained a simple expression for the minimum value of the zonal mean wind that allowed southward linear propagation in a quasigeostrophic $\beta$-plane flow when $\bar{v}$ $>0$.

The two effects of enhanced dissipation due to the Hadley circulation and the northward displacement of the critical layer into the westerly flow lead us to conclude that the reflection of planetary wave trains from the Tropics is unlikely in the winter hemisphere, at least at those longitudes where conditions do not greatly differ from the zonal mean. Of course, conditions in the real atmosphere are greatly complicated by further mixing due to transient waves, as well as by numerous other nonconservative effects, and GCM experiments with a realistic flow would be necessary to accurately quantify the importance of the Hadley cell in the planetary wave activity budget (see, e.g., Cook and Held 1992). Such experiments could also be used to investigate the summer hemisphere case, as a reasonable summer hemisphere basic state was not obtainable in our model using simple forcing of the form (2). ${ }^{5}$ The summer hemisphere problem is interesting as the meridional wind then allows linear propagation across easterly layers into the opposite hemisphere (e.g., Schneider and Watterson 1984; Watterson and Schneider 1987). Determining the extent to which this propagation can take place when the waves have finite amplitude is an important theoretical point that could further underpin our understanding of observed cross-hemispheric Rossby wave propagation (e.g., Tomas and Webster 1994). WPP showed that finite-amplitude waves can break even when there are weak westerlies at the equator, and it seems likely that the amplitude at which this breaking occurs would be strongly influenced by the direction and magnitude of the meridional winds at low latitudes.

Acknowledgments. This work was supported by the National Science Foundation, under Grant ATM9528471 to MIT and Grant ATM9527315 to Columbia University.

\section{REFERENCES}

Abramowitz, M., and I. E. Stegun, 1972: Handbook of Mathematical Functions. National Bureau of Standards, 106 pp.

Brunet, G., and P. H. Haynes, 1996: Low-latitude reflection of Rossby wave trains. J. Atmos. Sci., 53, 482-496.

Cook, K. H., and I. M. Held, 1992: The stationary response to large-

\footnotetext{
${ }^{5}$ However, one could take the approach of, for example, Schneider and Watterson (1984) and specify the desired flow, from which the forcing can then be calculated.
} 
scale orography in a general circulation model and a linear model. J. Atmos. Sci., 49, 525-539.

Farrell, B., and I. G. Watterson, 1985: Rossby waves in opposing currents. J. Atmos. Sci., 42, 1746-1755.

Frederiksen, J. S., and P. J. Webster, 1988: Alternative theories of atmospheric teleconnections and low-frequency fluctuations. Rev. Geophys., 26, 459-494.

Grose, W. L., and B. J. Hoskins, 1979: On the influence of orography on large-scale atmospheric flow. J. Atmos. Sci., 36, 223-234.

Hack, J. J., and R. Jacob, 1992: A global shallow water model based on the spectral transform method. NCAR Tech. Note NCAR/ TN-343+STR, $39 \mathrm{pp}$.

Haynes, P. H., 1988: Forced, dissipative generalizations of finiteamplitude wave-activity conservations for zonal and nonzonal basic flows. J. Atmos. Sci., 45, 2352-2362.

— 1989: The effect of barotropic instability on the nonlinear evolution of a Rossby wave critical layer. J. Fluid Mech., 207, 231266.

Held, I. M., and A. Y. Hou, 1980: Nonlinear axially symmetric circulations in a nearly inviscid atmosphere. J. Atmos. Sci., 37, $515-533$.

— , and P. J. Phillips, 1990: A barotropic model of the interaction between the Hadley cell and a Rossby wave. J. Atmos. Sci., 47, 856-869.

Horel, J. D., 1984: Complex principal component analysis: Theory and examples. J. Climate Appl. Meteor., 23, 1660-1673.

Hoskins, B. J., and D. J. Karoly, 1981: The steady linear response of a spherical atmosphere to thermal and orographic forcing. $J$. Atmos. Sci., 38, 1179-1196.

—, A. J. Simmons, and D. G. Andrews, 1977: Energy dispersion in a barotropic atmosphere. Quart. J. Roy. Meteor. Soc., 103, $553-567$.

Karoly, D. J., R. A. Plumb, and M. Ting, 1989: Examples of the horizontal propagation of quasi-stationary waves. J. Atmos. Sci. 46, 2802-2811.

Killworth, P. D., and M. E. McIntyre, 1985: Do Rossby-wave critical layers absorb, reflect or overreflect? J. Fluid Mech., 161, 449492.

Lindzen, R. S., and A. Y. Hou, 1988: Hadley circulations for zonally averaged heating centered off the equator. J. Atmos. Sci., 45, 2416-2427.

Magnusdottir, G., and P. H. Haynes, 1999: Reflection of planetary waves in three-dimensional tropospheric flows. J. Atmos. Sci., 56, 652-670.

Plumb, R. A., 1985: On the three-dimensional propagation of stationary waves. J. Atmos. Sci., 42, 217-229.

Polvani, L. M., J. G. Esler, and R. A. Plumb, 1999: Time variability and Simmons-Wallace-Branstator instability in a simple nonlinear one-layer model. J. Atmos. Sci., 56, 1445-1460.

Schneider, E. K., and I. G. Watterson, 1984: Stationary Rossby wave propagation through easterly layers. J. Atmos. Sci., 41, 20692083.

Simmons, A. J., J. M. Wallace, and G. W. Branstator, 1983: Barotropic wave propagation and instability, and atmospheric teleconnection patterns. J. Atmos. Sci., 40, 1363-1392.

Tomas, R. A., and P. J. Webster, 1994: Horizontal and vertical structure of cross-equatorial wave propagation. J. Atmos. Sci., 51, $1417-1430$.

Watterson, I. G., and E. K. Schneider, 1987: The effect of the Hadley circulation on the meridional propagation of stationary waves. Quart. J. Roy. Meteor. Soc., 113, 779-813.

Waugh, D. W., L. M. Polvani, and R. A. Plumb, 1994: Nonlinear, barotropic response to a localized topographic forcing: Formation of a "tropical surf zone" and its effect on interhemispheric propagation. J. Atmos. Sci., 51, 1401-1416.

Webster, P. J., 1981: Mechanisms determining the atmospheric response to sea surface temperature anomalies. J. Atmos. Sci., 38, $544-571$. 